\title{
Characterization of Turbulence Anisotropy, Coherence, and Intermittency at a Prospective Tidal Energy Site: Observational Data Analysis
}

\author{
Katherine McCaffrey ${ }^{\mathrm{a}, *}$ \\ Department of Atmospheric and Oceanic Sciences, University of Colorado at Boulder, \\ 216 UCB, CIRES Rm. 318, Boulder, CO 80309, USA \\ Baylor Fox-Kemper ${ }^{a, b}$, Peter E. Hamlington ${ }^{c}$, Jim Thomson ${ }^{d}$ \\ ${ }^{a}$ Cooperative Institute for Research in Environmental Sciences, University of Colorado at \\ Boulder, Boulder, CO \\ ${ }^{b}$ Department Earth, Environmental, and Planetary Sciences, Brown University, \\ Providence, $R I$ \\ ${ }^{c}$ Department of Mechanical Engineering, University of Colorado at Boulder, Boulder, CO \\ ${ }^{d}$ Applied Physics Laboratory, University of Washington, Seattle, WA
}

\section{Abstract}

As interest in marine renewable energy increases, observations are crucial for understanding the environments that prospective turbines will encounter. Data from an acoustic Doppler velocimeter in Puget Sound, WA are used to perform a detailed characterization of the turbulent flow encountered by a turbine in a tidal strait. Metrics such as turbulence intensity, structure functions, probability density functions, intermittency, coherent turbulence kinetic energy, anisotropy invariants, and a new scalar measure of anisotropy are used to characterize the turbulence. The results indicate that the scalar anisotropy magnitude can be used to identify and parameterize coherent,

\footnotetext{
* Corresponding author

Email address: katherine.mccaffrey@colorado.edu (Katherine McCaffrey)
} 
turbulent events in the flow. An analysis of the anisotropy characteristics leads to a physical description of turbulent stresses as being primarily oneor two-dimensional, in contrast to isotropic, three-dimensional turbulence. A new measure of the anisotropy magnitude is introduced to quantify the level of anisotropic, coherent turbulence in a coordinate-independent way. These diagnostics and results will be useful for improved realism in modeling the performance and loading of turbines in realistic ocean environments.

Keywords: Turbulence, Tidal Energy, Anisotropy, Coherence, Intermittency

\section{Introduction}

A full characterization of turbulence in the ocean would require observations in time and space spanning many orders of magnitude, which are unrealistic with the current technology. A large array of many instruments, sampling with high temporal frequency over a long period of time would be needed to sample an open-ocean location. Without such instrumentation, methods are needed to use the limited observing systems to extract as much temporal and spatial information as possible. In an energetic tidal channel, a single-point observing system such as an acoustic Doppler velocimeter (ADV), is able to sample with high temporal frequency, spanning the range of temporal scales most relevant to the location. In particular, an ADV is appropriate for stationary measurement locations, as in a tidal channel under consideration for a tidal energy conversion device, where only one location is needed for characterization (a vertical profile of ADVs would be ideal, to sample the entire water column, but measurements at a specifically chosen 
height can be sufficient for many purposes). Turbulence statistics developed for laboratory experiments (e.g. hot wire anemometers) or atmospheric boundary layer site assessment (e.g. sonic anemometers) can be adapted for use with these single-point observations in a tidal channel. This paper presents a number of such statistical analyses that shed light on the characteristic timescales and turbulent covariances, which can be used to estimate the dimensionality of the turbulent structures. Utilizing the "Frozen Turbulence Hypothesis" of Taylor [1], the length scales of the turbulent features can also be obtained from single-point observations.

Theoretical analyses have been used for studying the energy transfer and dissipation in tidal channels since Taylor [2]. Turbulence spectra were observed using a hot-film flowmeter towed behind a ship in a tidal channel by Grant et al. [3], showing an agreement between the theory of Kolmogorov [4] of an inertial range and the observations. The turbulent scales of motion that occur in an energetic tidal channel, such as the one studied here, are typically small coherent features (integral timescale $\mathcal{O}(10 \mathrm{~s})$, or $\mathcal{O}(10 \mathrm{~m})$ horizontal length scale) in comparison to the local depth (approximately $30 \mathrm{~m}$ ). However, occasional extreme events (autocorrelations persisting up to $150 \mathrm{~s}$ for $80 \mathrm{~m}$ horizontal scale) occur, which contribute to the turbulent energy spectrum. These turbulent features are not well-represented in coarseresolution coastal models [5] or statistical models (e.g., TurbSim Jonkman and Kilcher [6]), as will be shown in a forthcoming paper.

The use of an acoustic Doppler current profiler (ADCP) and a moored microstructure instrument by $\mathrm{Lu}$ et al. [7] allowed more of the turbulence spectrum to be observed, and they were able to estimate the production and 
dissipation rates of turbulent kinetic energy, as well as mixing length, eddy viscosity and diffusivity to assess and improve the turbulence parameterizations in a planetary boundary layer model. ADCP and ADV observations were used by Thomson et al. [8] to calculate turbulent dissipation rates in a tidal strait, with a critical method of removing the Doppler noise from the profiler data. These advances in observing systems have allowed for calculations of turbulent properties, and this paper utilizes these high-frequency observations for further analysis of the intermittent, coherent events that contribute to the turbulence spectrum.

Though a detailed turbulence characterization can serve many purposes, the one of interest here is marine renewable energy, and generating power from turbines placed in fast tidal currents, similar to wind power generation. The International Electrotechnical Commission (IEC) standard metric for quantifying the level of turbulence at wind energy sites is the turbulence intensity (addressed in Section 2.2) [9], but this metric does not address all turbulent events that may affect generation and safety, such as coherent structures and intense eddies. To date, studies of energetic tidal sites have continued to use the turbulence intensity as the primary metric for characterizing turbulent environments $[10,11,12,13,14]$. However, atmospheric and oceanic turbulence differ due to the distinct tidal, seasonal, and diurnal forcings of each fluid. Some of the most comprehensive characterizations, such as those described in Thomson et al. [15], Thomson et al. [16], and Gunawan et al. [17] have furthered the characterization of ocean turbulence by also examining energy spectra and spatial structure functions. Turbulence dissipation rates have also been measured in a small number of studies in 
the United Kingdom $[18,19]$. A more detailed description of energetic tidal channels using higher order statistics will reveal additional insights into the turbulent environment.

Turbulence manifested in gusts, or coherent, anisotropic, and intermittent eddies puts particularly strong and variable stresses on tidal turbines, leading to misalignment of the drive train and wearing of the gearbox (the specific structural make-up and design of the turbine will determine exactly the load, but the dynamic is the same as that for wind turbines) [20]. As a result, a site characterization that quantifies the incidence and properties of intense, coherent, anisotropic turbulent eddies has the potential to prevent untimely, unexpected, and costly failures in turbines. Anisotropic turbulence has been examined in theory [21,22], and in the laboratory [23], and observed in the form of mesoscale eddies in both the atmosphere [24] and the ocean $[25,26]$. In the present study, a higher-order, detailed characterization of ocean observations is performed that will provide more accurate information to numerical models that predict loading and power production from tidal turbines. These observations can be compared with numerical turbulence simulators (like the TurbSim model from the National Renewable Energy Laboratory (NREL) [6]), which currently use only turbulence intensity, a power spectral density curve, and model-defined spatial coherence to create more realistic turbulent environments for turbine simulator models (like the NREL FAST model).

Using velocity measurements from Puget Sound, WA, this paper examines several higher-order metrics to characterize and identify extreme turbulence eddies (or "events") in a tidal strait. These metrics include velocity 
structure functions for time scale information, probability density functions for intermittency, and anisotropy tensor eigenvalues for quantification and physical description of anisotropy. The specific metrics chosen are drawn from the laboratory and numerical experiment literature, where collection of data is simpler than in real-world ocean observations $[4,27,28]$. However, these metrics are demonstrated here to be suitable for application to observational data as well. Higher-order statistics and a parameterization more grounded in turbulence theory are proposed to improve the classification of anisotropy at potential tidal energy sites. An easily understood visualization of anisotropy proposed by Banerjee et al. [29] is presented as well. With an improved set of parameters that provide a better physical description of the flow, it will be shown that more accurate predictions of turbulence coherence can be obtained. Knowledge of turbulence coherence will allow turbines to be better designed to withstand the particular scales of turbulence that cause the largest loads and put the most strain on gear boxes. This paper is organized as follows: Section 2 characterizes the flow with several statistical parameters; Section 3 proposes a new parameterization based on the characterization; and Section 4 concludes with a discussion of the implications of this work to the marine energy industry, and to observations of turbulence in the ocean generally.

\section{Characterization of Turbulence}

The data used in this analysis were collected from an acoustic Doppler velocimeter (ADV) device at Nodule Point, on the eastern side of Marrowstone Island in the Puget Sound [15]. The site, which is $22 \mathrm{~m}$ deep, was under 
consideration for an array of Verdant Power ${ }^{\mathrm{TM}}$ turbines and has a maximum current velocity of $1.8 \mathrm{~m} \mathrm{~s}^{-1}$ at the proposed hub-height of $4.7 \mathrm{~m}$ above the seabed. The measurements examined here were collected from February 17th to February 21, 2011 using an ADV sampling at $32 \mathrm{~Hz}$ on the apex of a Tidal Turbulence Tripod at approximately hub-height. The location is well-mixed, with minimal stratification as measured from a conductivity-temperaturedepth (CTD) sensor. More detail on the observations and how they were performed can be found in Thomson et al. [15].

\subsection{Velocity Decomposition and Statistics}

Each of the three velocity components have been decomposed into a mean $(\overline{\mathbf{u}})$ and perturbation $\left(\mathbf{u}^{\prime}\right)$ :

$$
\mathbf{u}=\overline{\mathbf{u}}+\mathbf{u}^{\prime},
$$

where $\mathbf{u}=u \mathbf{i}+v \mathbf{j}+w \mathbf{k}$. The horizontal velocities are defined where $-\mathbf{i}$ is aligned toward the seaward principal flow direction, $\mathbf{j}$ is perpendicular to the principal flow direction, and $\mathbf{k}$ is in the vertical direction. Wave motions are neglected for the location analyzed here because the orbital effects do not reach the depth of the turbine, although they may be important in other locations since they introduce coherent structures that appear in the variance. Though recent work suggests that some wave effects may penetrate deeper [30, 31, 32], Thomson et al. [33] show that waves in Puget Sound typically have a $3 s$ period, because they are fetch limited, giving them about a $15 \mathrm{~m}$ wavelength, and thus most motion has decayed $7.5 \mathrm{~m}$ below the surface. The ADV measurements are $16.3 \mathrm{~m}$ below the surface, which is more than twice the e-folding depth from the wave layer. Thus, we also do 
not explicitly consider deep-penetrating wave-forced (i.e. Langmuir) turbulence, though it may affect turbulence at other sites [32, 34].The mean used here is a 10-minute time mean, by contrast to the shorter 5 -minute mean chosen by Thomson et al. [15]. The 10-minute interval was chosen in an attempt to retain the longest timescales of coherent turbulence structures in the perturbation, $u^{\prime}$, while capturing the tidal and diurnal variations in the mean, $\bar{u}$. Within each averaging interval, the mean flow was assumed to be steady, which can create discontinuities at interval edges. Experimentation with a range of averaging intervals suggested that 5 to 10 minute variability might be appropriately categorized as turbulent, although all coherent structures observed passed by in less than 4 minutes. (see Appendix). Assuming Taylor's frozen turbulence hypothesis [1], this allows motions smaller than $\sim 1.1 \mathrm{~km}$ at Nodule Point (for $1.8 \mathrm{~m} \mathrm{~s}^{-1}$ mean velocities).

Figure 1 shows the three components of velocity at Nodule Point from 17 Feb 2011 to 21 Feb 2011 at approximately hub-height depth $(4.7 m)$, with the observed velocities in gray, and the 10-minute mean in black. "Slack conditions," where the velocity is not large enough to drive a turbine, are defined as $\bar{u} \leq 0.8 \mathrm{~m} \mathrm{~s}^{-1}$ (shown with dotted lines on Figure 1), and occur at high and low tides. This is in contrast to ebb and flood tides when velocities are larger. The analyses here will focus on flood and ebb events (distinguished by being upstream and downstream of headland) consistent with the emphasis on tidal power generation. Velocity perturbations, the individual components of turbulent kinetic energy $\left(T K E=\frac{1}{2}\left(\overline{u^{\prime 2}}+\overline{v^{\prime 2}}+\overline{w^{\prime 2}}\right)\right)$, and total $T K E$ are shown in Figures 2a, b, and c. Peaks occur periodically in each signal with the M2 (semidiurnal) tide dominated mainly by the $\overline{u^{\prime 2}}$ and $\overline{v^{\prime 2}}$ 
components of the TKE. Diurnal variability is also typical as one large and one small (i.e., mixed semi-diurnal) flood or ebb per day. The $w^{\prime}$ fluctuations are considerably smaller than those in the other directions, as indicated by the small $\overline{w^{\prime 2}}$ component of the TKE.

Reynolds shear stresses are defined as $\overline{u_{i}^{\prime} u_{j}^{\prime}}$. In a well-mixed, homogeneous flow such as a tidal strait, coherent turbulent structures appear as bursts in the Reynolds shear stresses [35]. These may be formed by geographical or topographical features that disturb the otherwise-laminar tidal flow. Figure 2c shows the covariances, where turbulent bursts can be seen occurring at a roughly diurnal period. Outside of the turbulent burst, all three components are approximately zero with only occasional peaks in one component. Some days have two turbulent bursts, both during flood tide, with the stronger turbulence associated with the stronger flood.

\subsection{Turbulence Intensity, Turbulent Kinetic Energy, and Coherent Turbu- lent Kinetic Energy}

The metric most commonly used in the wind industry to characterize the turbulent environment is the turbulence intensity, $I$ [36]. Turbulence intensity, shown in Figure 3a, is the ratio of the standard deviation of the velocity to the mean with a noise-corrected term subtracted for acoustic Doppler measurements, and is defined as

$$
I_{u}=\frac{\sigma_{u}}{\bar{u}}=\frac{\sqrt{\overline{u^{\prime 2}}-n^{2}}}{\bar{u}},
$$

where the overline indicates a 10-minute average $\left(I_{u} \approx \frac{\sqrt{\frac{2}{3} T K E-n^{2}}}{\bar{u}}\right.$ for isotropic turbulence) [15]. Although in much of the wind literature turbulence intensity is calculated from wind speeds (often measured by cup anemometers) 
a) $U$ velocity

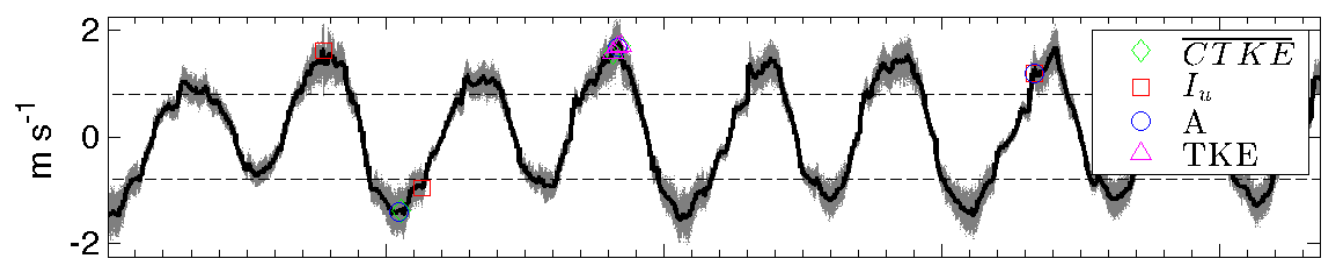

b) $\vee$ velocity

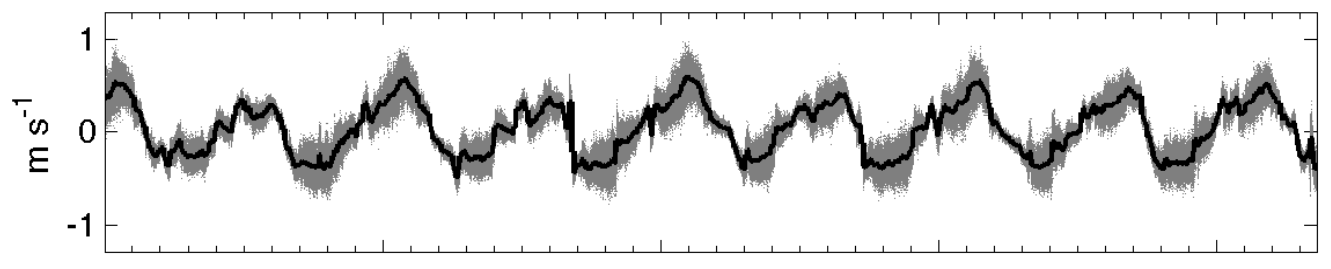

c) W velocity

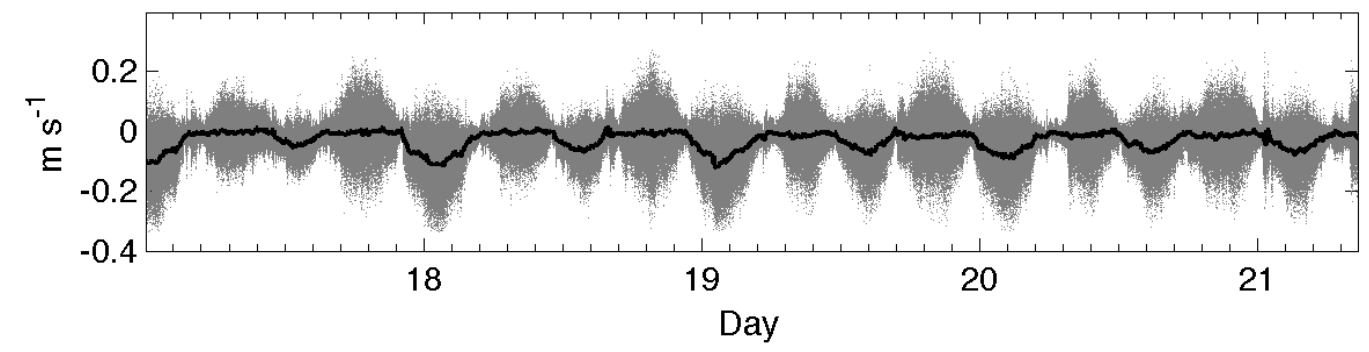

Figure 1: Velocities in the along stream (u; a), cross-stream (v; b), and vertical (w; c) directions from the ADV at Nodule Point from 17 Feb 2011 to 21 Feb 2011 at approximate hub-height depth of $4.7 \mathrm{~m}$. Gray dots show instantaneous velocity measurements, and black lines show 10-minute averages. Dotted lines in a) show the slack condition criterion. Three 99th percentile values of $I_{u}, C T K E$, and $A$ values when $\bar{u}>0.8 \mathrm{~m} \mathrm{~s}^{-1}$ are shown in the red squares, green diamonds, and blue circles, respectively. 
a) Velocity Perturbations

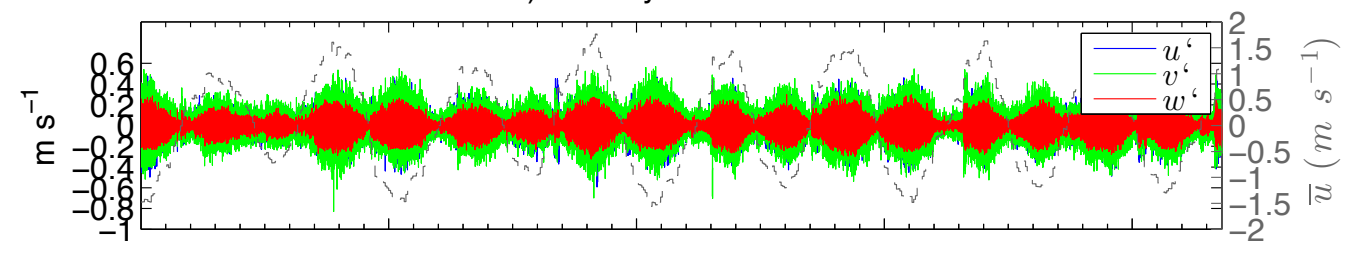

b) Normal Stresses

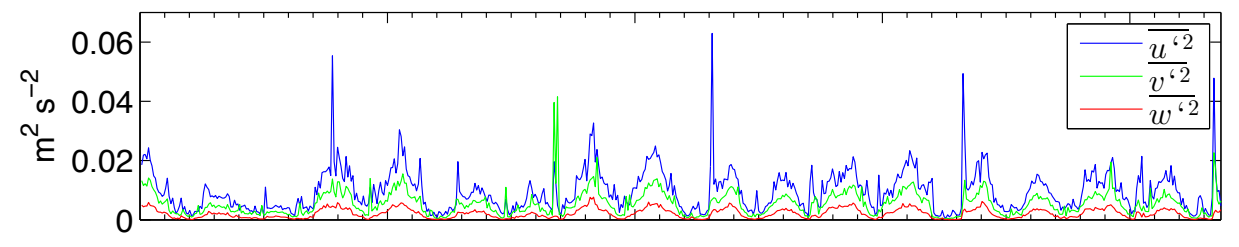

c) Shear Stresses

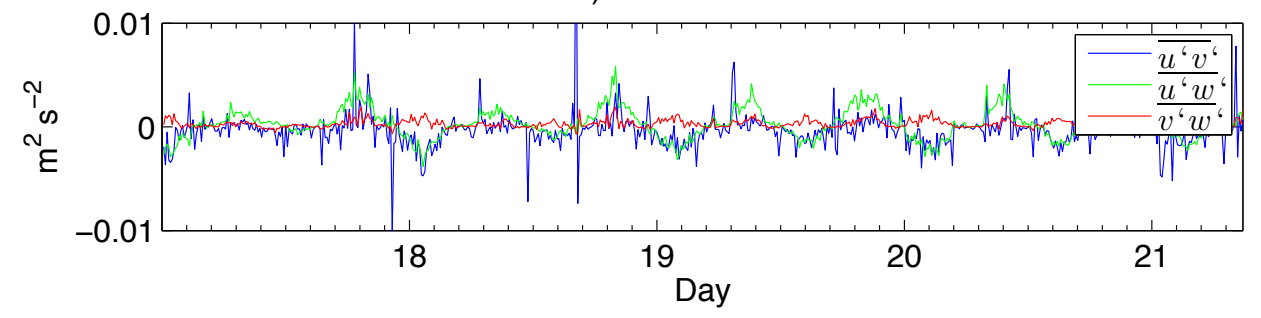

Figure 2: a) Velocity perturbations, b) turbulent kinetic energy components, and c) Reynolds shear stress components, from the Nodule Point ADV shown in Figure 1. 
a) $I_{u}$

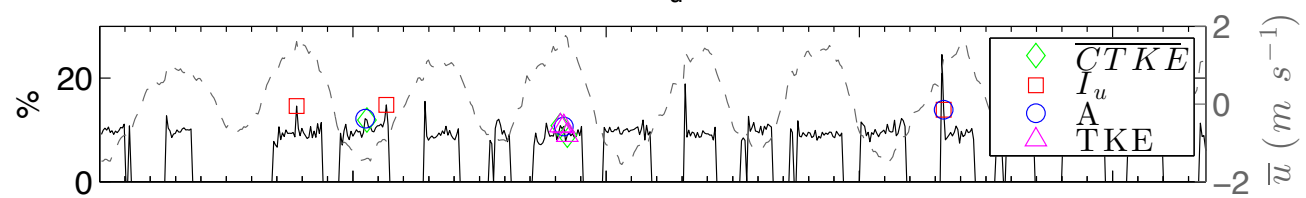

b) TKE

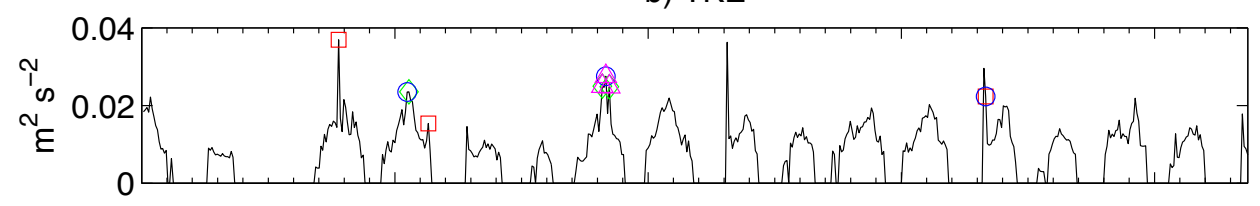

c) $\overline{C T K E}$

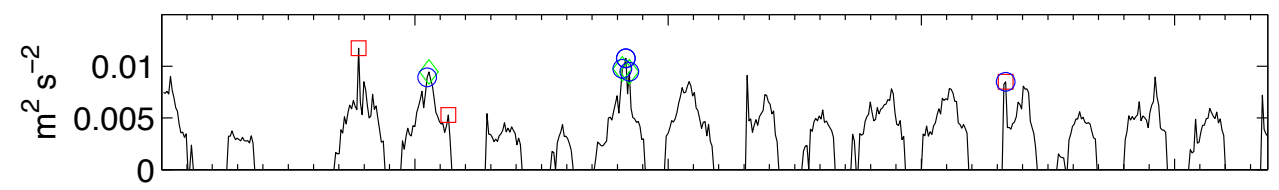

d) $A$

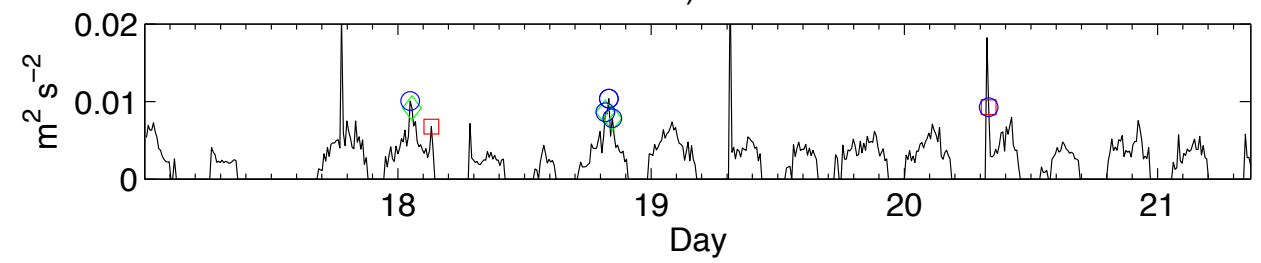

Figure 3: a) Turbulence intensity, b) turbulent kinetic energy, c) coherent turbulent kinetic energy, and d) anisotropy magnitude, $A$, from the Nodule Point data shown in Figure 1, with intervals with $\bar{u}>0.8 \mathrm{~m} \mathrm{~s}^{-1}$ removed. Three 99th-percentile values of $I_{u}, T K E, \overline{C T K E}$, and $A$ values when $\bar{u}>$ $0.8 \mathrm{~m} \mathrm{~s}^{-1}$ are shown in the red squares, green diamonds, and blue circles, respectively, discussed in Sections 2.2 and 2.6. The mean $u$-velocity is shown in a) as the gray dashed line. 
[37], turbulence intensity can be calculated in all three directions $\left(I_{u}, I_{v}, I_{w}\right)$, but the along-stream intensity, $I_{u}$, is used here. Figure 4 shows turbulence intensity plotted versus mean flow speed for the entire sampling period at Nodule Point. The highest turbulence intensities occur below $0.8 \mathrm{~m} \mathrm{~s}^{-1}$, which are considered slack conditions when the turbine would be motionless. The fastest mean velocities at Nodule Point $\left(\sim 2 \mathrm{~m} \mathrm{~s}^{-1}\right)$ see turbulence intensities around $10 \%$, while slower mean velocities see turbulence intensities that reach up to $20 \%$. This behavior is consistent with the $I_{u} \sim 1 / \bar{u}$ relationship, which has been regressed in the inset of Figure 4 where $\overline{u^{\prime 2}}$ vs. $\bar{u}$ is shown. Similarly, MacEnri et al. [38] saw the same behavior, but with lower overall turbulence intensity levels in the Strangford Lough, Ireland. This variation in $I_{u}$ encourages further analysis into what causes the turbulence intensity to peak, since the large spread suggests that local values of $\bar{u}$ are not a good predictor of $\sigma_{u}$.

$I_{u}$ only accounts for one direction of velocity fluctuations, so turbulent kinetic energy, $T K E$, is also used for a more complete characterization of tidal turbulence intensity. TKE is defined as one-half the sum of the normal stresses,

$$
T K E=\frac{\overline{u_{i} u_{i}}}{2}=\frac{1}{2}\left(\overline{u^{\prime 2}}+\overline{v^{\prime 2}}+\overline{w^{\prime 2}}\right),
$$

has also been shown have a negative impact on power production [39]. Although turbulence intensity and $T K E$ are a helpful metrics for determining loads on a turbine [40] and expected energy production, a more detailed characterization of the type of turbulence will show individual turbulent events like a large, anisotropic eddy passing through the region (peaks in figure $3 \mathrm{~b}$ ). Coherent turbulent kinetic energy (CTKE; the magnitude of the instanta- 
neous Reynolds shear stresses) is another common metric in the wind literature used to identify coherent turbulent events [10, 41], and is defined as

$$
C T K E=\frac{1}{2} \sqrt{\left(u^{\prime} v^{\prime}\right)^{2}+\left(u^{\prime} w^{\prime}\right)^{2}+\left(v^{\prime} w^{\prime}\right)^{2}} .
$$

$C T K E$ identifies the instances when the Reynolds shear stresses peak, while the turbulence intensity identifies only one component of the kinetic energy. The use of the cross terms identifies the moments when there are peaks in multiple velocity components, identified as spatially coherent features in the flow (though $C T K E$ is an instantaneous quantity, so the temporal coherence remains unknown). The reader is referred to Kelley et al. [35], where CTKE is introduced, for more information on this metric.

The method employed by Kelley et al. [35] \& [42] to describe coherent turbulent structures uses wavelet analysis to decompose the Reynolds stresses and coherent turbulent kinetic energy alongside observed loads on wind turbines to characterize the time and frequency behavior of the coherent structures and their effect on the turbines. These results have shown that bursts of CTKE induce higher structural loads at scales $6 \%-23 \%$ of the rotor diameter on both stiff and flexible-blade wind turbines. Although observations of loads are not available for a tidal turbine (loads have been measured in a flume tank [43, 44] but not in the field), Reynolds stresses, $T K E$, and $C T K E$ from ADV measurements can be used to infer expected loading events.

$C T K E$ is shown in Figure 3c, and will be used as an additional metric for parameterization of turbulence at this location. An alternative to $C T K E$, the anisotropy magnitude $A$ (Eq. 12), that is more firmly grounded in tur- 
bulence theory is shown in Figure 3d and presented in Section 2.6. From Figure 3, it is possible to identify more "events," appearing as peaks in the bottom three panels that do not appear in the turbulence intensity. Three illustrative intervals in the largest 99th percentile of $I_{u}, T K E, \overline{C T K E}$, and $A$ when $\bar{u}>0.8 \mathrm{~m} \mathrm{~s}^{-1}$ were chosen to highlight the meaning of these diagnostics to draw attention to where the peaks in each metric lies. These intervals are also indicated in Figures 1, 3, 4, 8, 9, and 10.

\subsection{Correlation and Length Scales}

Turbulence intensity does not directly address the spatial and temporal structure of turbulence in the tidal channel, so quantifying the scales of motion leading to large $I_{u}$ is a natural next step in the characterization. Spatial and temporal correlation scales can also give a much better physical description of the turbulence than is possible with turbulence intensity. Thus, velocity autocorrelations were calculated to infer the time (and, using Taylor's hypothesis, length) scales of the turbulence. The temporal autocorrelation is defined as

$$
\rho(\tau)=\frac{\overline{u^{\prime}(t) u^{\prime}(t+\tau)}}{\overline{u^{\prime 2}}}
$$

where the overbar is the 10-minute mean, and results are shown in Figure 5. The coherence function used in TurbSim is also plotted (with $a=8.8, L=$ 2.454.7, $f=0.0001$ Jonkman [41]), showing the stretched exponential form used to include coherent structures in the model. Although the model significantly under predicts the coherence for certain intervals, it does capture the mean observed at this location quite well.

The Taylor, $\lambda$, and integral, $\Lambda$, scales are used to quantify the longest time over which the turbulence stays correlated, and the time until the flow 


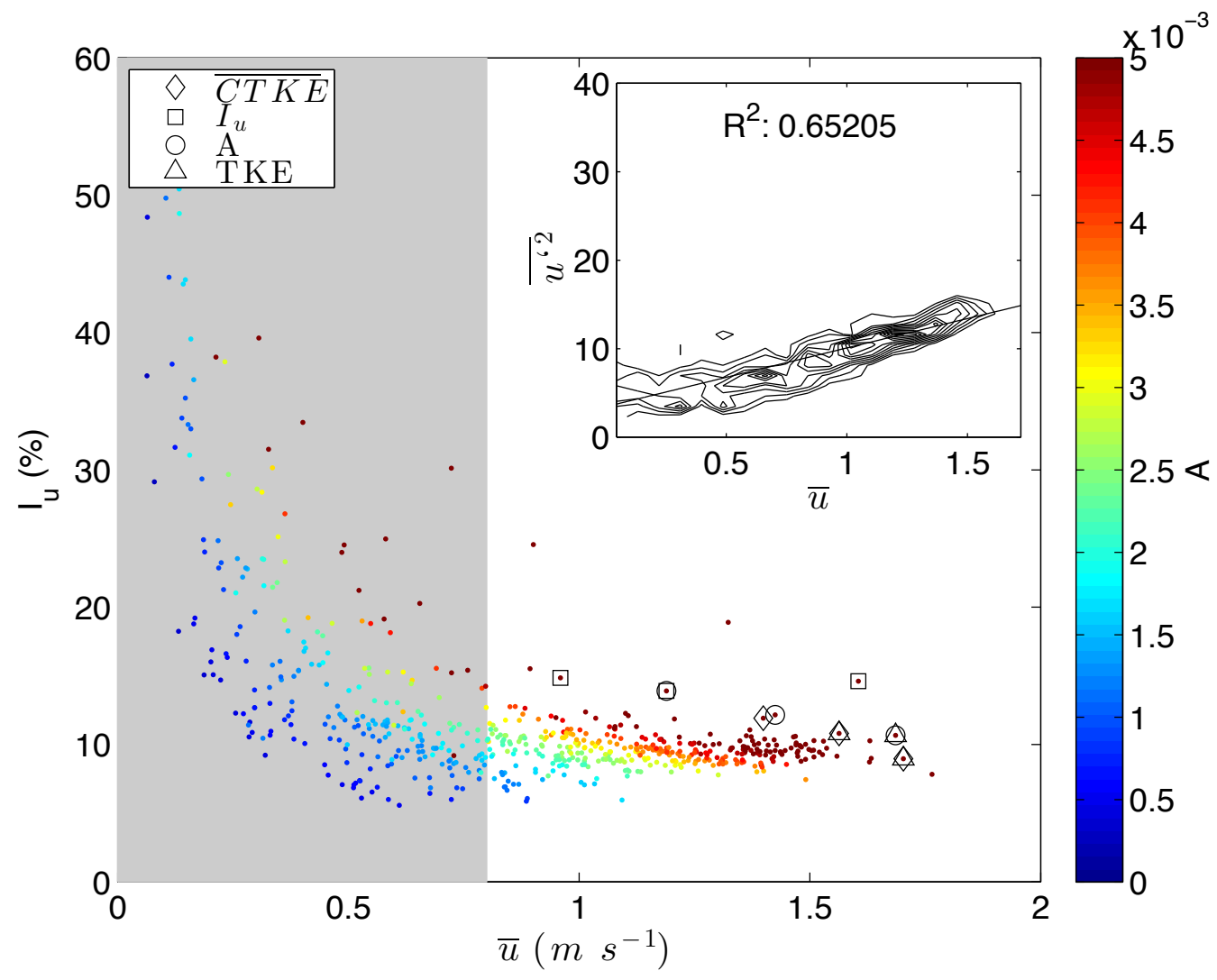

Figure 4: Turbulence intensity versus mean speed for each 10-minute interval at Nodule Point, colored by $A$, discussed in Section 2.6. The gray area indicates slack conditions. Three 99th percentile values of $I_{u}, T K E, C T K E$, and $A$ values when $\bar{u}>0.8 \mathrm{~m} \mathrm{~s}^{-1}$ are shown in the squares, triangles, diamonds, and circles, respectively on each plot. Inset is the joint pdf of $\bar{u}$ vs $\overline{u^{\prime 2}}$, with the best-fit line. 
is uncorrelated, respectively [45]. These scales are defined as

$$
\begin{array}{r}
\lambda^{2}=-2\left[\left.\frac{d^{2} \rho}{d \tau^{2}}\right|_{\tau=0}\right]^{-1}, \\
\Lambda=\int_{0}^{\tau_{0}} \rho(\tau) d \tau,
\end{array}
$$

where $\tau_{0}$ is the first zero-crossing of the autocorrelation function, eliminating the residual noise from the un-averaged random variable. These correlation time scales were calculated for each 10 -minute interval where $\bar{u}>0.8 \mathrm{~m} \mathrm{~s}^{-1}$ in Figure 5. The average correlation length scales are shown, though Figure 5 clearly shows intervals with much higher $\lambda$ and $\Lambda$ than the mean. Using Taylor's frozen turbulence hypothesis with the mean horizontal velocity and $\Lambda$ for each 10-min segment, the average correlation length scale is $11.6 \mathrm{~m}$, with the longest correlation equal to $81 \mathrm{~m}$, equivalent to the average $\Lambda=10 \mathrm{~s}$ and longest $\Lambda=70 \mathrm{~s}$. Thomson et al. [15] calculated a dominant length scale of $2-3$ times the water depth (average of $75 \mathrm{~m}$ ) at Nodule Point from the "fractional" turbulence intensity, with a large spread. The fractional turbulence intensity is based on the energy spectrum, which is dominated by the large scales, possibly causing the difference between scales observed by the different methods. The correlation scales presented here identify the most common scales, not the most energetic ones, which are identified by the fractional turbulence intensity. Features with a length scale larger than the water depth are inherently anisotropic, as isotropy can only exist up to the length scale of the water depth.

\subsection{Temporal Structure Functions}

Structure functions of each 10-minute interval with $\bar{u}>0.8 \mathrm{~m} \mathrm{~s}^{-1}$ were computed to study the relationship of correlation over the longer and shorter 

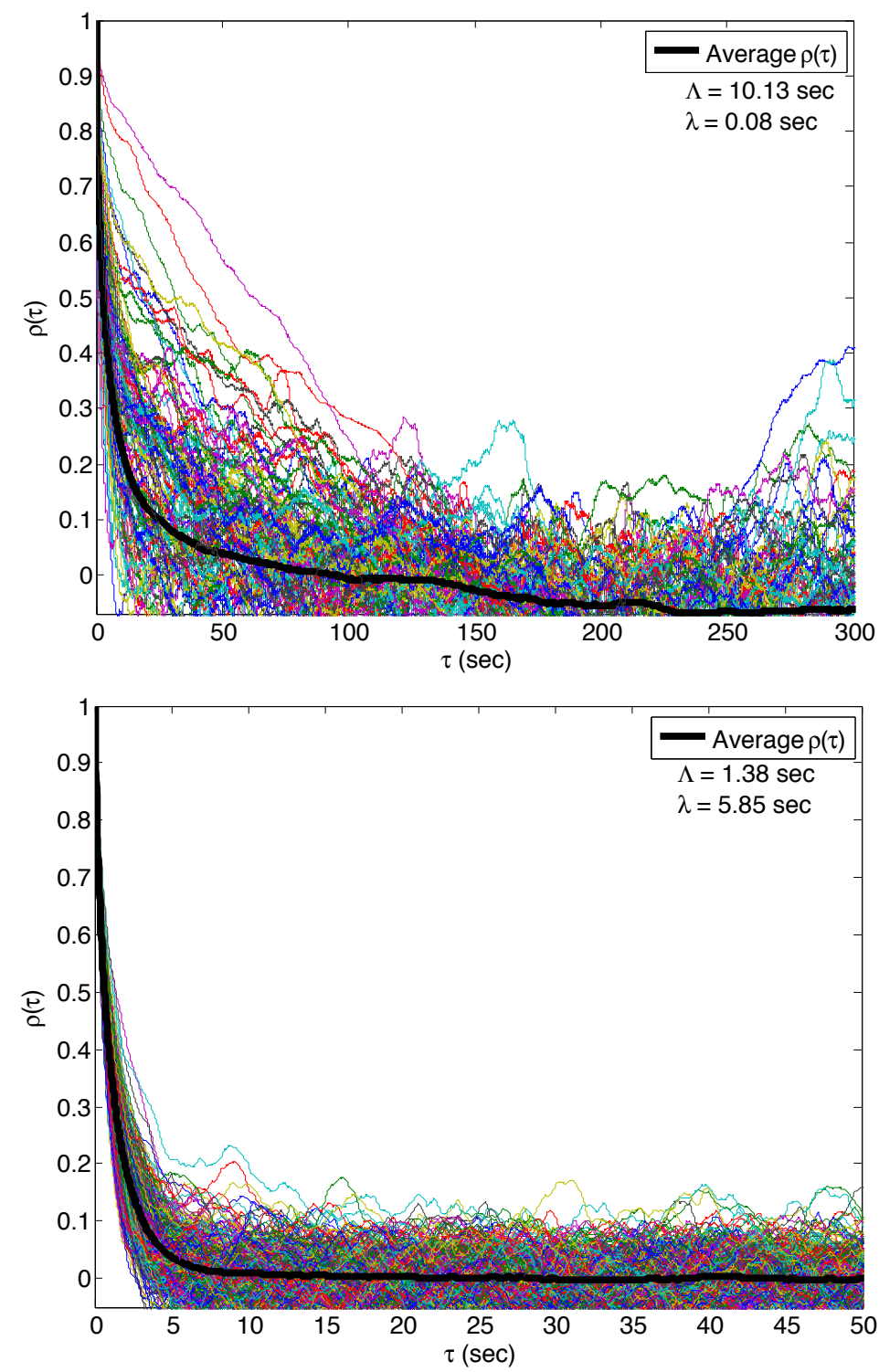

Figure 5: Autocorrelation functions of horizontal (top) and vertical (bottom) velocity for a subsample of 10-minute intervals with $\bar{u}>0.8 \mathrm{~m} \mathrm{~s}^{-1}$ from the Nodule Point ADV, with the average autocorrelation shown in the bold solid line and the average integral and Taylor scales, $\Lambda$ and $\lambda$. 
timescales, analogous to an energy spectrum. Structure functions are especially useful for problems with uneven measurements in space or in time, but they are used here with the even time-series of observations to directly relate to the correlation time scales from the previous section. The second-order temporal structure function is defined as [46]

$$
D(\tau, t)=\overline{[u(t)-u(t+\tau)]^{2}},
$$

which has a slope $\left(\gamma_{D}\right)$ that is related to the slope of the energy spectrum $\left(\gamma_{E}\right)$ by

$$
\gamma_{D}=-\gamma_{E}-1
$$

This relationship can be used to define the energy spectrum of simulated turbulence used as input into a computational fluid model of a turbine. Structure functions provide spectral information about a range of timescales, as opposed to $\lambda$ and $\Lambda$ which provide single correlation scales. Spatial structure functions have also been used to infer energy dissipation rates, as done in Wiles et al. [47], Thomson et al. [15] and Lucas et al. [48].

Figure 6 compares the temporal structure function of the horizontal (top) and vertical (bottom) velocities from the Nodule Point ADV. For easier comparison, the solid line in the horizontal structure function plot is the median of the vertical structure functions, and vice versa. The slope of nearly $\gamma_{D}=2 / 3$ seen in both directions (shallower in the horizontal) up to a time scale of approximately $3 s$ matches the scaling theory of three-dimensional, isotropic turbulence from Kolmogorov [4]. It is important to note that the smaller scales span a larger range in structure function amplitudes, so though the average slope is shallower than $\gamma_{D}=2 / 3$, there are many intervals with 
steeper slopes than average. The structure function slope of $\gamma_{D}=2 / 3$ is equivalent to the frequency spectral slope of $f^{-5 / 3}$. The structure function median in one direction is almost always outside of the 25 th percentile of the other direction's structure function (see solid lines on each plot), exhibiting the preference for larger horizontal velocity covariance at these time scales.

At first glance, this difference in amplitude between the vertical and horizontal velocity structure functions seems to suggest that the covariances are not isotropic, but McCaffrey et al. [49] show that the transformation from frequency to temporal space introduces an integration constant that may explain the offset in structure function amplitude seen here. Thus, only the slope of the structure function is meaningful in this context, not the magnitude. A slight flattening occurs on the vertical structure function at about $3 \mathrm{~s}$ that does not occur in the horizontal structure function. This supports the results of Thomson et al. [15] for frequency spectra with 5-minute windows, which exhibit isotropy (horizontal and vertical spectra having the same slope) from $1-10 s$ scales. At lower frequencies, the vertical spectra flatten out, showing the large-scale anisotropy. A random phase signal has a structure function slope of 0, suggesting that over the longest timescales vertical velocities are not associated with a turbulent cascade. Note that the $\gamma_{D}=2 / 3$ slope of these structure functions (and the related kinetic energy spectrum slope) is not a unique indicator of an energy cascade in three-dimensional turbulence -indeed the inverse energy cascade in two-dimensional turbulence of Kraichnan [50] would also follow $\gamma_{D}=2 / 3$, so the extension of the horizontal spectral slope to larger scales is not inconsistent with correlation scales of 10s. Consistency of the structure function with $\Lambda$ is indicated by the point 
where the structure function flattens out being on the order of the average integral scale from Figure 5. The vertical correlation scales seen in Figure 5 are also consistent with the results, since they average about $1 s$.

\subsection{Intermittency from Probability Density Functions}

The flow diagnosis reveals distinctions among different 10-minute intervals, which likely extends to turbulence intermittency. A useful tool for analyzing turbulent behavior is the velocity difference probability density function (pdf), which can quantify the intermittency of turbulence (e.g. coherent structures and eddies). Comparing pdfs in each direction further uncovers the anisotropy. Gaussian turbulence should manifest itself as a collection of velocity differences that are normally distributed for small differences, with intermittent events appearing as deviations from Gaussian distributions in the tails [51]. Although the small velocity differences in all pdfs shown in Figure 7 do follow the Gaussian curve (dashed lines), the strong departure from Gaussian in the tails indicates that there is a significant amount of intermittency in this flow.

Higher statistical moments were calculated for each velocity component (subscript $i$ ) also in 10-minute intervals (mean, variance, skewness $S$, and kurtosis $K$, respectively) to provide more quantitative measures of intermittency. Moments of the u-velocity at Nodule Point are plotted in Figure 8. A purely random, Gaussian flow would have moments $S_{i}=0$ and $K_{i}=3$. It is clear from the skewness and kurtosis (shown here as deviations from Gaussian, $K-3$ ) that many intervals deviate from normally distributed velocity perturbations (for consistency across moments, the Doppler noise was not re-

moved from the standard deviation as it was for turbulence intensity). The 

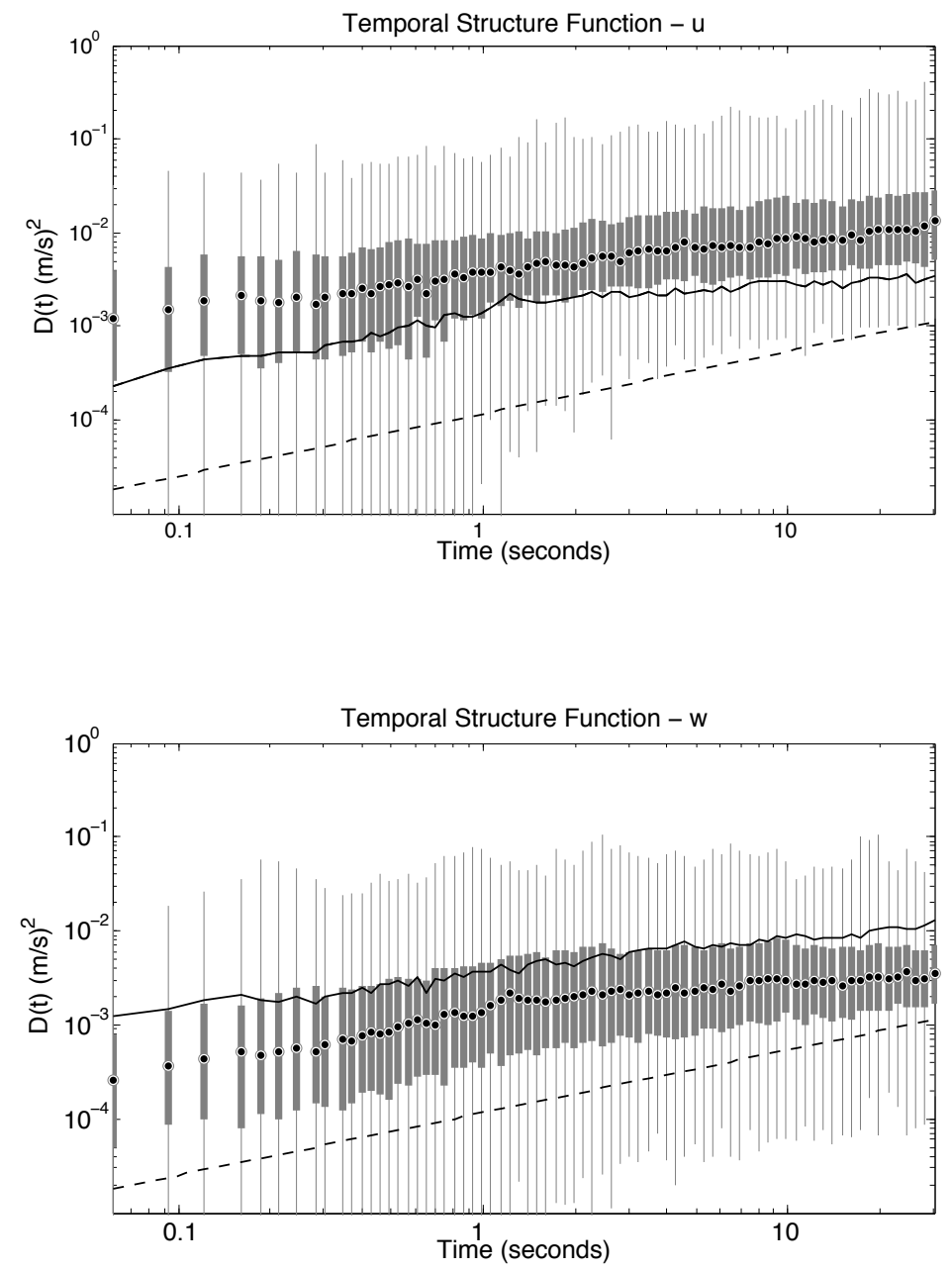

Figure 6: Box plots of the second-order temporal structure functions for each 10-minute interval with an $\bar{u}>0.8 \mathrm{~m} \mathrm{~s}^{-1}$ of the Nodule Point ADV data. Top plot is the horizontal velocity structure function and the bottom is the vertical velocity structure function. For comparison, the solid line on the horizontal structure functions shows the median of the vertical velocity structure functions, and the line on the vertical structure functions shows the median of the horizontal velocity structure functions. White circles are the median, the boxes show the 25th-percentile, and the vertical lines include the entire range. The dotted line is a guideline with slope of $2 / 3$. 
departure from Gaussian in the pdfs expands consistently on the departure from 0 in the skewness and kurtosis (minus 3) in Figure 8.

A single observation at Nodule Point covers $1 / 32$ of a second, so looking at velocity differences over slightly longer intervals will accentuate persistent features. Figure 7 compares the pdfs using $\Delta t=1 / 32 s, 3.6 s$ and $7.2 s$, corresponding to the smallest features captured by the ADV, and at $3 \mathrm{~m}$ and $6 m$, which are half- and full-rotor diameters for the Verdant Power turbines, assuming Taylor's hypothesis (with the overall average of the flow speed as the velocity scale). As the time interval increases, the intermittency increases for $u$ and $v$ velocities but decreases for $w$. Thus, as in the structure function analysis, horizontal velocities are more coherent on longer timescales than vertical, and now we may associate those correlations as well with intermittency. There is only a small difference in intermittency between the 3 and $6 m$-scales, suggesting that the intermittent structures occur on a scale larger than the turbine rotor in this location. Other deeper locations that can accommodate larger turbines may also contain longer turbulence scales, so though these observations are not relevant to all locations, the measurement technique is useful.

\subsection{Tensor Invariant Anisotropy Magnitude}

The metrics analyzed thus far indicate that turbulence at this location in the Puget Sound features anisotropy and coherence. However CTKE is, like $I_{u}$, not a true scalar as it depends on the coordinate system chosen. In the case of $C T K E$, the dependence on coordinate system is not obvious, but direct calculation using this data and alternative coordinate system choices, such as a rotation of the u-v coordinates by $45^{\circ}$, results in an altered 


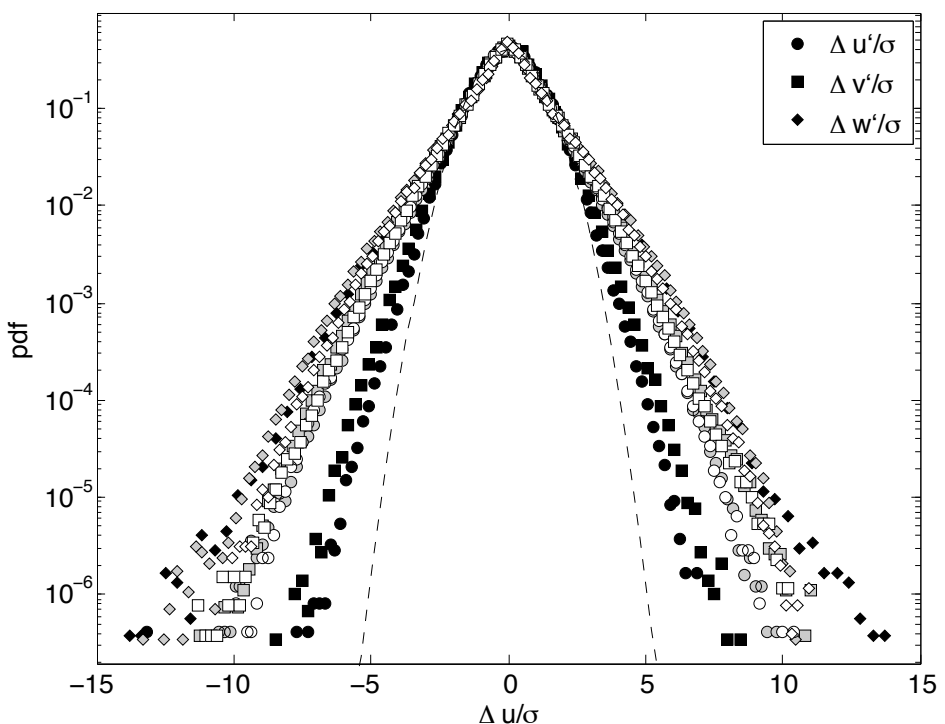

Figure 7: Probability density functions of the velocity perturbation differences, $\Delta u^{\prime}$ (circles), $\Delta v^{\prime}$ (squares), and $\Delta w^{\prime}$ (diamonds) from Nodule Point ADV data, with Gaussian curves for reference (dashed). Black shapes have a time step of $\Delta t=1 / 32 s(\sim 3 \mathrm{~cm})$, gray are $\Delta t=3.6 \mathrm{~s}(\sim 3 \mathrm{~m})$, and white are $\Delta t=7.2 s(\sim 6 m)$. 

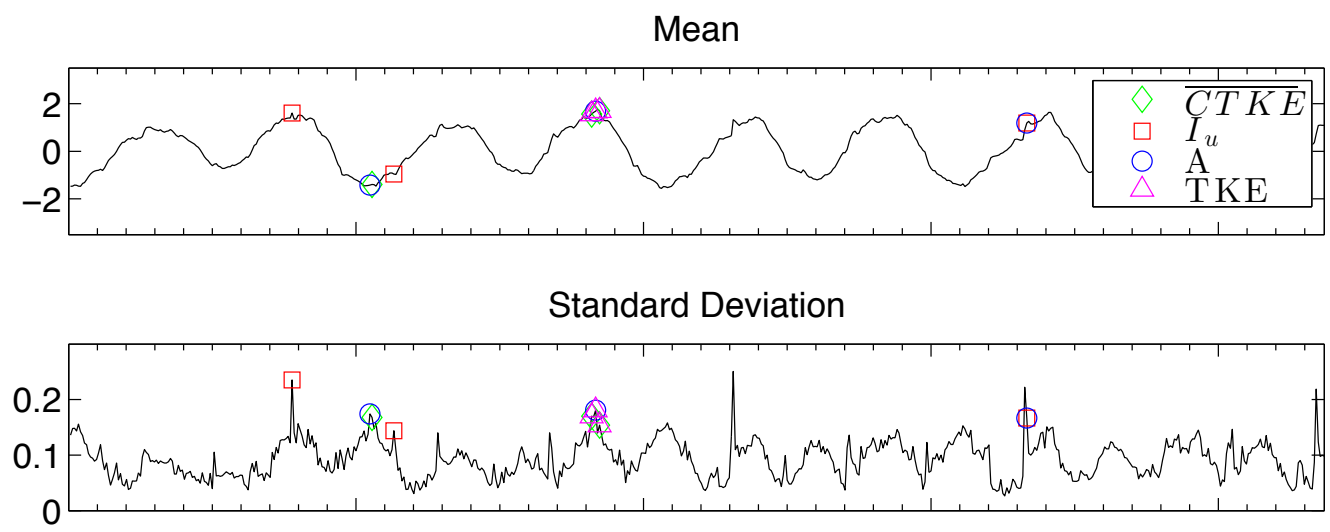

Skewness
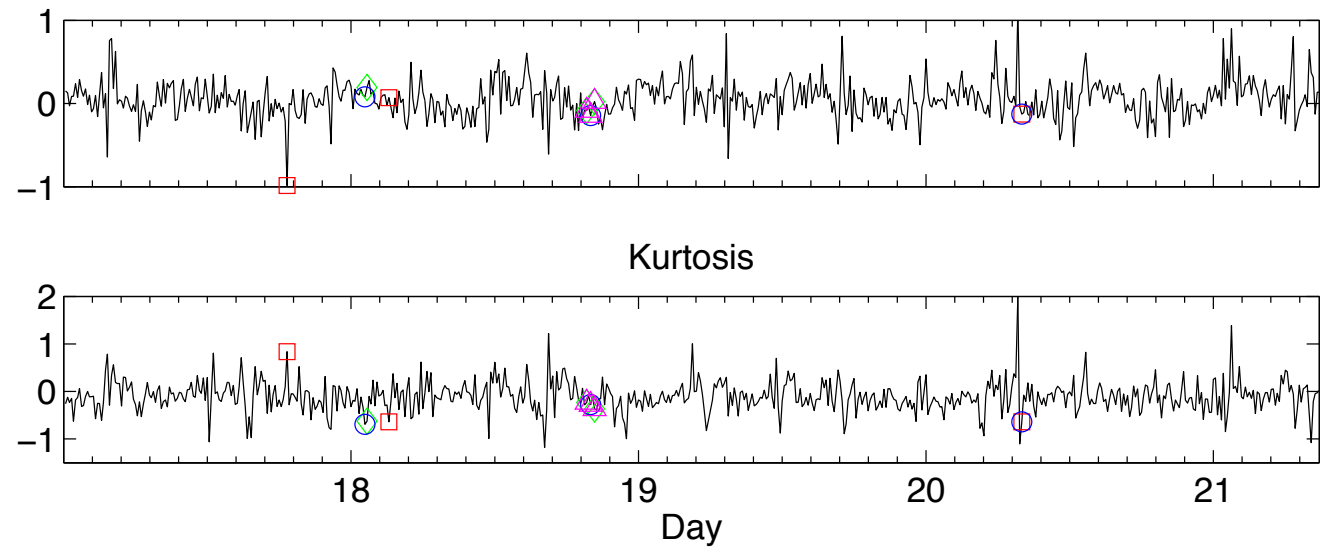

Figure 8: First four moments of the u-velocity from the Nodule Point ADV data, based on 10-minute intervals. Top-bottom: mean, standard deviation, $\sigma$, skewness, $S$, and kurtosis minus $3, K-3$. Three 99th-percentile values of $I_{u}, T K E, \overline{C T K E}$, and $A$ values when $\bar{u}>0.8 \mathrm{~m} \mathrm{~s}^{-1}$ are shown in the red squares, magenta triangles, green diamonds, and blue circles, respectively. 
value of $C T K E$. By aligning the $u$-velocity with the along-stream direction and $w$ with the vertical as done here, one arrives at a unique definition of $C T K E$. However, the turbulent structures that arrive at the study location may observe coordinate-invariance symmetries not shared by $C T K E$ (e.g., non-horizontal planar or axial symmetries). Locations where the ebb and flood flow directions are not anti-parallel are likely to cause inconsistencies in the interpretation of $C T K E$. Ideally, a true scalar, invariant of coordinate system, could be used to quantify turbulent events. TKE is tensor invariant, but does not describe the anisotropy between its components, or include the shear stresses. To this end, we created a new metric which we call the anisotropy magnitude, $A$, that captures the shear stress terms like the $C T K E$, but also the anisotropy from the normal stresses as well as the strength of the turbulence in the $T K E$.

The flow at Nodule Point has been determined to be anisotropic and to possess intermittent coherent structures. A coordinate-system-invariant metric of anisotropy in the presence of such structures is therefore desirable. A more detailed anisotropy analysis can be done using the anisotropy tensor $[52]:$

$$
a_{i j}=\frac{\overline{u_{i} u_{j}}}{2 T K E}-\frac{\delta_{i j}}{3}, T K E=\frac{\overline{u_{i} u_{i}}}{2}
$$

and its three principal invariants $(I, I I, I I I)$,

$$
\begin{aligned}
I & =a_{i i} \equiv 0 \\
I I & =a_{i j} a_{j i} \\
I I I & =a_{i j} a_{i n} a_{j n},
\end{aligned}
$$

with a sum implied to occur over repeated indices (Einstein notation). Like 
the turbulent kinetic energy, $T K E$, these invariants are true scalars independent of coordinate system-they depend only on the symmetry, or lack thereof, of the turbulence itself. An analysis of the invariants $I I$ and $I I I$ (since $I=0$ by the definition of $a_{i j}$ ) provides a method of quantifying anisotropy in a turbulent flow and physically describing the departures from isotropy, independent of the chosen coordinate axis. Isotropic turbulence with uncorrelated orthogonal velocity fluctuations has the characteristic that $I=I I=I I I=0$, and deviations away from this point describe different turbulent regimes, as illustrated by the classical Lumley Triangle [28]. The next section illustrates an updated version of the Lumley Triangle due to Banerjee et al. [29].

The coordinate-system invariant scalar magnitude of the anisotropy similar to the $C T K E$, denoted $A$, is constructed from the scalars $I I$ and $T K E$ as

$$
\begin{aligned}
& A=T K E \sqrt{I I} \equiv \sqrt{\frac{1}{2}\left({\overline{u^{\prime} v^{\prime}}}^{2}+{\overline{u^{\prime} w^{\prime}}}^{2}+{\overline{v^{\prime} w^{\prime}}}^{2}\right)+\frac{1}{2} k-\frac{k^{2}}{3}} \\
& \frac{\sqrt{\frac{1}{2}\left({\overline{u^{\prime} v^{\prime}}}^{2}+{\overline{u^{\prime} w^{\prime}}}^{2}+{\overline{v^{\prime} w^{\prime}}}^{2}\right)+\frac{1}{4}\left(\overline{u^{\prime 2}}+\overline{v^{\prime 2}}+\overline{w^{\prime 2}}\right)-}}{\frac{1}{12}\left({\overline{u^{\prime 2}}}^{2}+{\overline{v^{\prime 2}}}^{2}+{\overline{w^{\prime 2}}}^{2}\right)-\frac{1}{6}\left(\overline{u^{\prime 2}} \overline{v^{\prime 2}}+\overline{v^{\prime 2}} \overline{w^{\prime 2}}+\overline{v^{\prime 2}} \overline{w^{\prime 2}}\right) .}
\end{aligned}
$$

This definition possesses the following attributes: 1) Unlike $T K E$, but like $C T K E, A=0$ for isotropic, uncorrelated turbulence. 2) Like CTKE and $T K E, A$ has the units of energy per unit mass $\left(m^{2} s^{-2}\right)$. 3) $A$ tends to grow with $C T K E$, approaching a version of $C T K E$ formed from the time-averaged shear stresses as $C T K E \gg T K E$. 4) Like $C T K E$ and $T K E, A$ is real, and 5) unlike $C T K E, A$ is a true, coordinate-independent scalar, as it is the product of two scalars. The anisotropy magnitude, $A$, is therefore similar in meaning to the $C T K E$ in Equation 4, though not identical because $C T K E$ is 
an instantaneous measure, and $A$ uses the 10-minute means of the Reynolds stresses. This allows instrument noise to be removed through the averaging, while it acts to enhance $C T K E$. The anisotropy magnitude, $A$, is plotted at Nodule Point in Figure 3c, exhibiting simultaneous, similar intermittent peaks as $C T K E$ between periods of low anisotropy. However, it is intriguing to note that the extreme occurrences of $T K E$ co-occur with two peaks in $C T K E$, and one in $A$, but the peaks in $A$ do not always co-occur with those of $C T K E$ or $I_{u}$. This highlights the fact that neither $C T K E$ nor $I_{u}$ are reliable, coordinate-system independent indicators of turbulence structure or anisotropy, but that $T K E$ and $A$ capture the same features in a reliable, coordinate-system independent manner.

\subsection{Anisotropic Barycentric Map}

Banerjee et al. [29] introduce a visualization of anisotropy that contains additional information beyond $A$, based on the eigenvalues of the anisotropy tensor as opposed to the invariants. The "barycentric map" is a ternary diagram with vertices representing purely one-component (linear), two-component (planar), and three-component (isotropic) turbulence. This map is easier to read than the Lumley Triangle since the three turbulent states are equally spaced, and each have one point to represent them. The Cayley-Hamilton theorem proves that the eigenvalues may be found using only the tensor invariants $(I, I I, I I I)$ and vice versa. Thus like the invariants, the eigenvalues (and the barycentric map) are coordinate-independent [53]. The axisymmetric and plane-strain limits are represented by straight lines on the barycentric map.

If the eigenvalues of the anisotropy tensor, $a_{i j}$ are $\lambda_{1}, \lambda_{2}$ and $\lambda_{3}$, where 
$\lambda_{1} \geq \lambda_{2} \geq \lambda_{3}$, the coordinates of the barycentric map are

$$
\begin{aligned}
& C_{1 c}=\lambda_{1}-\lambda_{2}, \\
& C_{2 c}=2\left(\lambda_{2}-\lambda_{3}\right), \\
& C_{3 c}=3 \lambda_{3}+1 .
\end{aligned}
$$

To plot on a Cartesian plane where the vertices of the barycentric map are $\left(x_{1 c}, y_{1 c}\right),\left(x_{2 c}, y_{2 c}\right)$, and $\left(x_{3 c}, y_{3 c}\right)$, the location of each point is

$$
\begin{gathered}
x_{\text {new }}=C_{1 c} x_{1 c}+C_{2 c} x_{2 c}+C_{3 c} x_{3 c} \\
y_{\text {new }}=C_{1 c} y_{1 c}+C_{2 c} y_{2 c}+C_{3 c} y_{3 c}
\end{gathered}
$$

The barycentric map is shown in Figure 9, with four color schemes to be discussed in Section 3: turbulence intensity, $I_{u}, C T K E, A$, and TKE. These results show that the vast majority of the flow is in the middle of the map, extending closer to the one- and two-component limits. The large empty space on the top part of the triangle highlights that this flow is never in the isotropic turbulent regime for scales between the sampling frequency and the 10-minute window.

The eigenvectors of the anisotropy tensor, when ordered, give the principal axes of the turbulence. In the one-component limit, the eigenvector associated with the largest eigenvalue orients the (linear) direction of the flow, and the plane made by the eigenvectors of the two largest eigenvalues describes the two-component turbulence. Here, the linear turbulence is in the along-flow direction, and the plane is the horizontal plane in the alongand cross-flow directions. It is also possible to gain the directional information from Figure $2 \mathrm{~b}$ and $2 \mathrm{c}$ to see which of the Reynolds stress components 


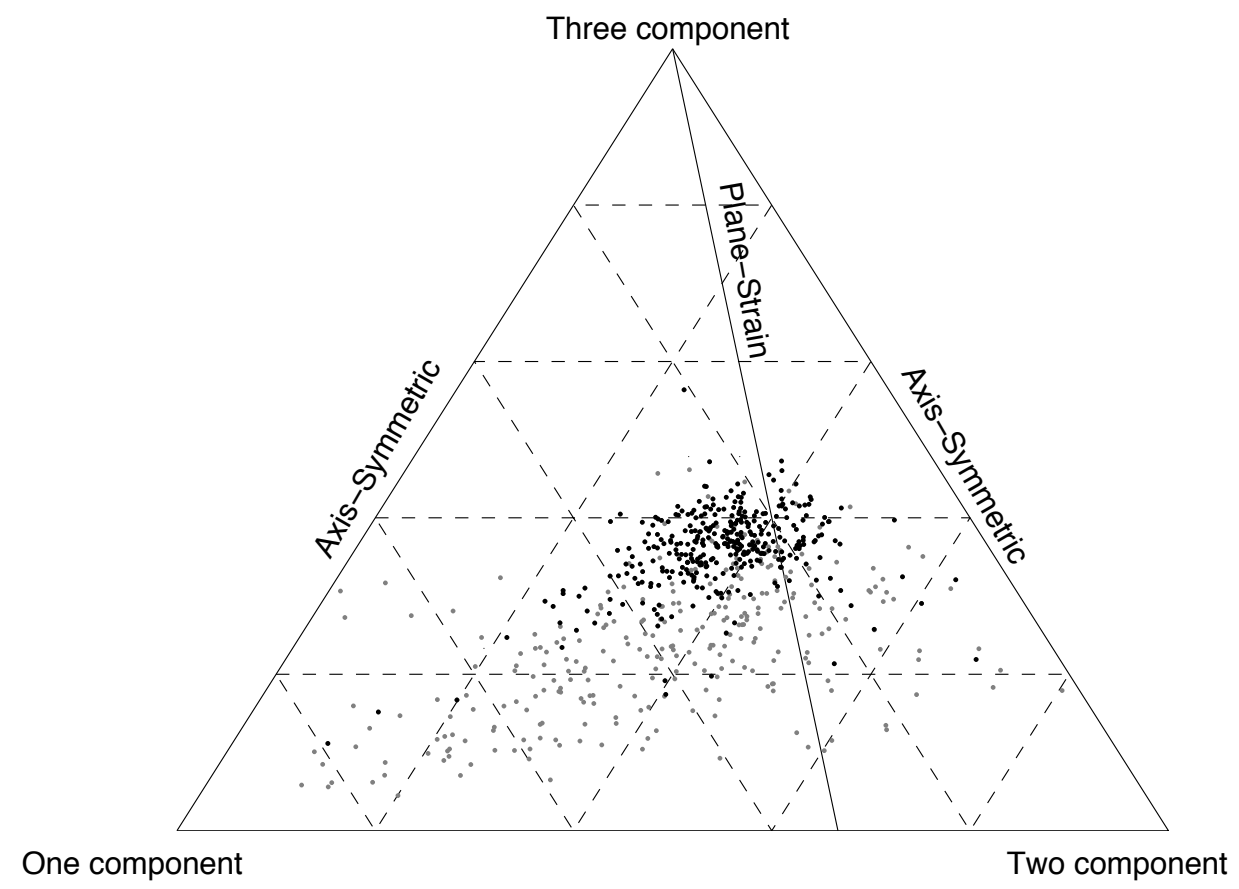

Figure 9: Barycentric maps based on $C_{1 c}, C_{2 c}$, and $C_{3 c}$ for the 10-minute intervals with $\bar{u}>0.8 \mathrm{~ms}^{-1}$ at Nodule Point. The black points are the intervals with $\bar{u}>0.8 \mathrm{~m} \mathrm{~s}^{-1}$, and the gray points are intervals falling under "slack tide" conditions.

dominates the $C T K E$ signal. Consistency between these approaches derives from the close relationship between the Reynolds stresses and the anisotropy tensor.

\section{Parameterization of Extreme Turbulence}

A single parameter, or small set of parameters, to describe the turbulence at a particular location is desired for modeling and classification of tidal turbine locations. The turbulence intensity has been the parameter traditionally 
used by engineers, and only briefly has CTKE been introduced to discuss coherence in a flow $[10,11,12,35]$. Here, the metrics discussed in Section 2 are compared to identify the best parameter to characterize turbulence.

A comparison of the joint probabilities of $I_{u}, T K E, \overline{C T K E}$, and $A$ is a first step to showing how one parameter can be used in place of a long list. Table 1 and 2 show the percentage of intervals (with $\bar{u}>0.8 \mathrm{~ms}^{-1}$ ) that fall above the 90th- and 99th-percentile for each pair of variables. Since the probability of the turbulence intensity peaking when any of the other metrics is also peaking is below $10 \%$ when compared to TKE and CTKE (and only slightly higher than $10 \%$ for $A$ ), this shows that there is no statistically significant correlation between the extreme values of the metrics, and the metrics are therefore capturing different features of the flow. On the other hand, the probabilities of intervals falling above the 90th- and 99thpercentiles of all other pairs of metrics is above $50 \%$, and often above $75 \%$, indicating that the metrics are capturing the majority of the same events, and do not all need to be calculated. Focussing on the comparison between $A$ and $C T K E$ (Figure 10), for example, illustrates how one metric can replace the use of two.

There is a strong correlation $\left(R^{2}=.66\right)$ between $A$ and $\overline{C T K E}$ (since $A$ was derived to be the invariant form of $C T K E$ ), and the highest $I_{u}, T K E$, $\overline{C T K E}$ and $A$ intervals all appear in the high end of Figure 10. This supports the use of $A$ as opposed to $\overline{C T K E}$ in characterizing intense turbulent events since it similarly captures anisotropic, coherent events, but is a tensor invariant quantity better supported by turbulence theory than $\overline{C T K E}$. Since $C T K E$ has been demonstrated to correlate with turbine loading, and 


\begin{tabular}{c|c|c|c|c} 
& $A$ & $I_{u}$ & $T K E$ & $\overline{C T K E}$ \\
\hline$A$ & 100 & 12.7 & 76.2 & 73.0 \\
\hline$I_{u}$ & 12.7 & 100 & 6.4 & 6.4 \\
\hline$T K E$ & 76.2 & 6.4 & 100 & 96.8 \\
\hline$\overline{C T K E}$ & 73.0 & 6.4 & 96.8 & 100
\end{tabular}

Table 1: Percentage of intervals with $\bar{u}>0.8 \mathrm{~m} \mathrm{~s}^{-1}$ that, if they are in the 90th-percentile of one metric, they also fall in the 90th-percentile of the other. A random probability would expect this value to be $10 \%$.

$A$ is closely related, it is a natural supposition that $A$ would have the same impact, but further studies must be conducted to determine whether the $\sqrt{I I}$ (anisotropic turbulence) or the TKE (intense turbulence) factors of coherent turbulence, or the combination of both, measured by $A$ are most closely related to loading.

The turbulence intensity versus mean speed plot gains a great deal of information when colored based on $A$, as in Figure 4. The $1 / u$ behavior is expected from the definition of $I_{u}$, and the scatter in the points is informative, but color based on $A$ highlights the points that will have the most impact on the turbine - with the highest $I_{u}$ when the mean speed is highest. The 99th-percentile intervals of $I_{u}, T K E, \overline{C T K E}$, and $A$ when $\bar{u}>0.8 \mathrm{~m} \mathrm{~s}^{-1}$ all occur in this region as well. Figures 10 and 4 both support the use of $A$ over $C T K E$ and $I_{u}$, but a parameterization based on the physical properties of the flow is the greater goal of this work.

Using $A$ as a measure of coherence, supported by Figure 12, and compar- 


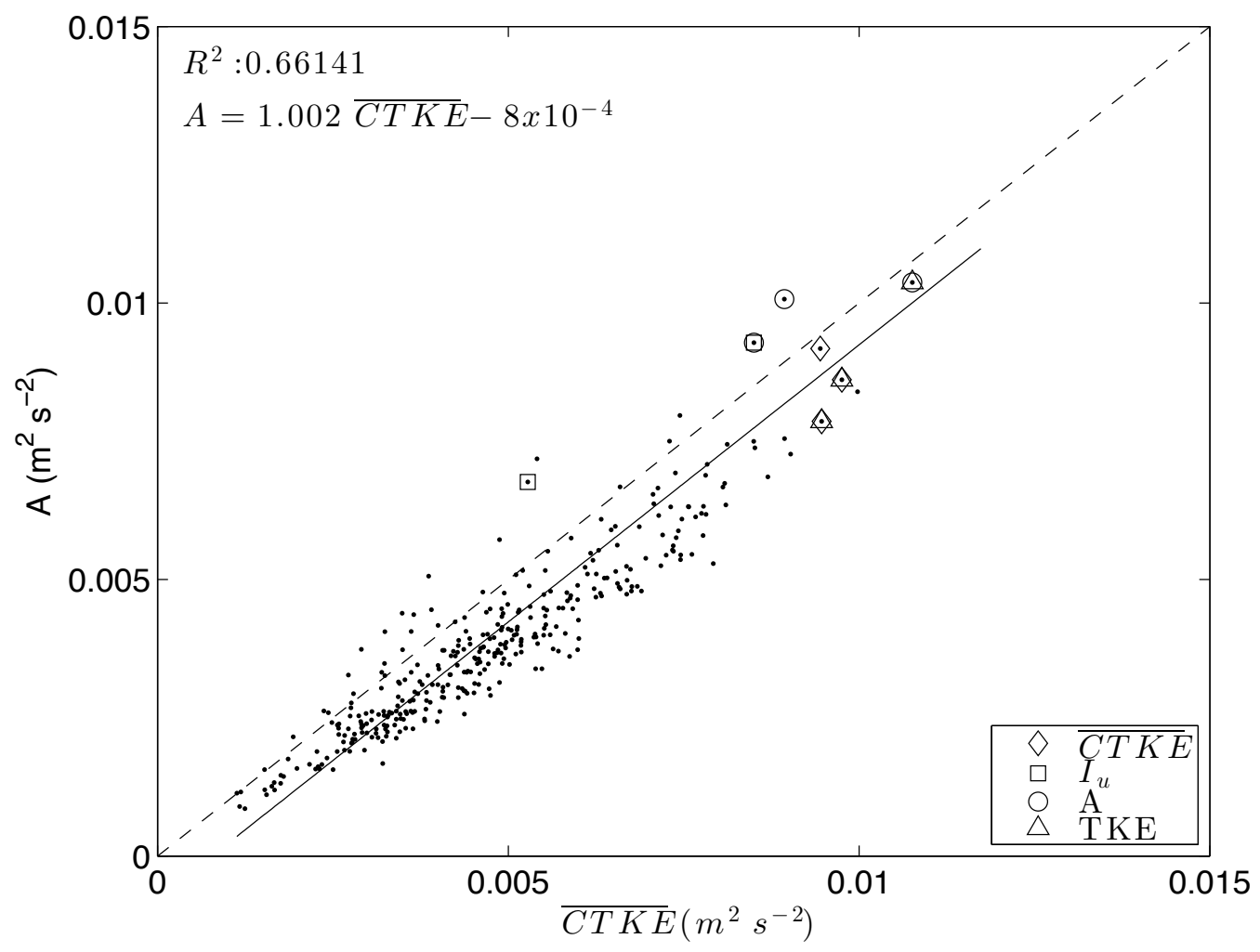

Figure 10: $A$ versus mean $C T K E$ for each 10-minute interval at Nodule Point. Linear best-fit and correlation coefficient are shown in the solid line, and the one-to-one dashed line is shown as well. Three 99th-percentile values of $I_{u}, T K E, \overline{C T K E}$, and $A$ values are shown in the squares, triangles, diamonds, and circles, respectively. 


\begin{tabular}{c|c|c|c|c} 
& $A$ & $I_{u}$ & $T K E$ & $\overline{C T K E}$ \\
\hline$A$ & 100 & 0 & 83.3 & 50.0 \\
\hline$I_{u}$ & 0 & 100 & 0 & 0 \\
\hline$T K E$ & 83.3 & 0 & 100 & 66.7 \\
\hline$\overline{C T K E}$ & 50.0 & 0 & 66.7 & 100
\end{tabular}

Table 2: Percentage of intervals with $\bar{u}>0.8 \mathrm{~m} \mathrm{~s}^{-1}$ that, if they are in the 99th-percentile of one metric, they also fall in the 99th-percentile of the other. A random probability would expect this value to be $1 \%$.

ing the larger $A$ events $\left(A>0.005 \mathrm{~m}^{2} \mathrm{~s}^{-2}\right)$ to the total flow on a probability density function, the higher $A$ events appear as smaller deviations from Gaussian in the tails (see Figure 11). Although slightly counter-intuitive, this result suggests that within a single coherent structure, velocity increments are more Gaussian, but in differences spanning from one smaller-scale structure to the next, the intermittency appears. These results show that the coherent events at $\Delta t=7.2 \mathrm{~s}(\Delta x \sim 6 \mathrm{~m})$ are nearly random in all three directions, as is expected from the structure function analysis and the spectral results of Thomson et al. [15], which are isotropic at this scale. It is possible that using the pdf to parameterize intermittency with $A$ is not useful, since choosing $\Delta t$ may be a larger indicator of intermittency, so the dependence on $A$ does not appear. Again, the anisotropy seen in Figure 7 appears in the pdf differences in the three directions.

Parameterizing coherence with $I_{u}, T K E, \overline{C T K E}$, and $A$ is possible with regression analysis between each of these metrics and the integral and Taylor 


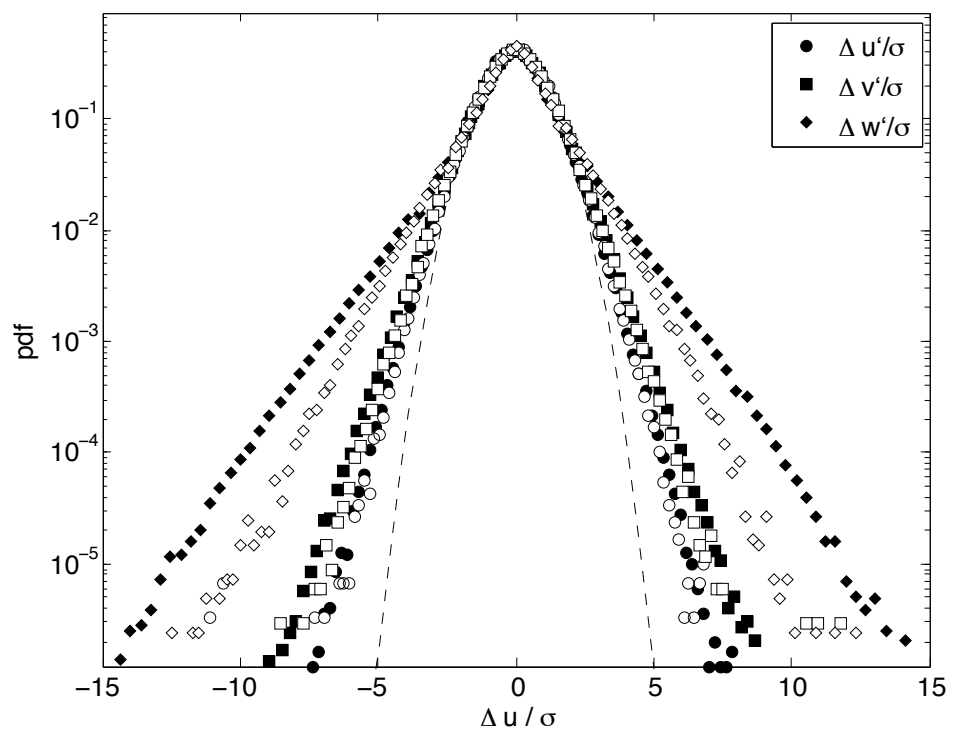

Figure 11: Probability density functions of the velocity perturbation differences, $\Delta u^{\prime}$ (circles), $\Delta v^{\prime}$ (squares), and $\Delta w^{\prime}$ (diamonds) with a $\Delta t=$ $7.2 s(\sim 6 m)$ from Nodule Point ADV data, with Gaussian curves for reference (dashed). Black includes when $A<0.005 \mathrm{~m}^{2} \mathrm{~s}^{-2}$, white includes when $A>0.005 m^{2} s^{-2}$. 
scales, $\Lambda$ and $\lambda$. Table 3 shows these results, highlighting that the intervals with high $I_{u}, T K E, A$, and $\overline{C T K E}$ are associated with longer turbulent timescales $\lambda$ and $\Lambda$. A most closely parameterizes coherence in the Taylor scale, $\lambda$, while the coefficients of determination are lower for TKE, $\overline{C T K E}$ and $I_{u}$ (i.e., poorly predicted). Correlation is low between the four metrics and the Integral scale, $\Lambda$. Quadratic regressions between $I_{u}$, and $\overline{C T K E}$ (most often used for this purpose; Jonkman [41]) and $\lambda$ or $\Lambda$ do not yield statistically significant coefficients of determination (i.e., poor predictability). Thus, if we consider our long turbulent timescales to indicate coherent structures, then the extremal intervals do indicate the presence of coherent structures, but the metrics $I_{u}, T K E$, and $\overline{C T K E}$ are not only a measure of coherence. However, the quadratic relationship between $A$ and the Taylor scale, $\lambda$ is strong, with an $R^{2}$ value of 0.884 (TKE also has a high correlation, though it is exceeded by $A$ ). This relationship is plotted in Figure 12. $C T K E$ and $A$ include more information about cross-correlations and directional variability than the turbulence intensity, so it is perhaps not surprising that they give a better physical description of the flow and a less noisy prediction of other measures of coherence in the turbulence, which is the goal of this study. The advantage of turbulence intensity over CTKE and $A$ is that directional velocity observations are not needed, only a current speed. However, without directional information, $I_{u}$ is a poor predictor of coherence, and one should consider adding other measures of coherence, such as $\lambda$, in addition to $I_{u}$.

Anisotropy is possibly the most important feature of the turbulence to parameterize since it is not captured at all by the currently-used turbulence 


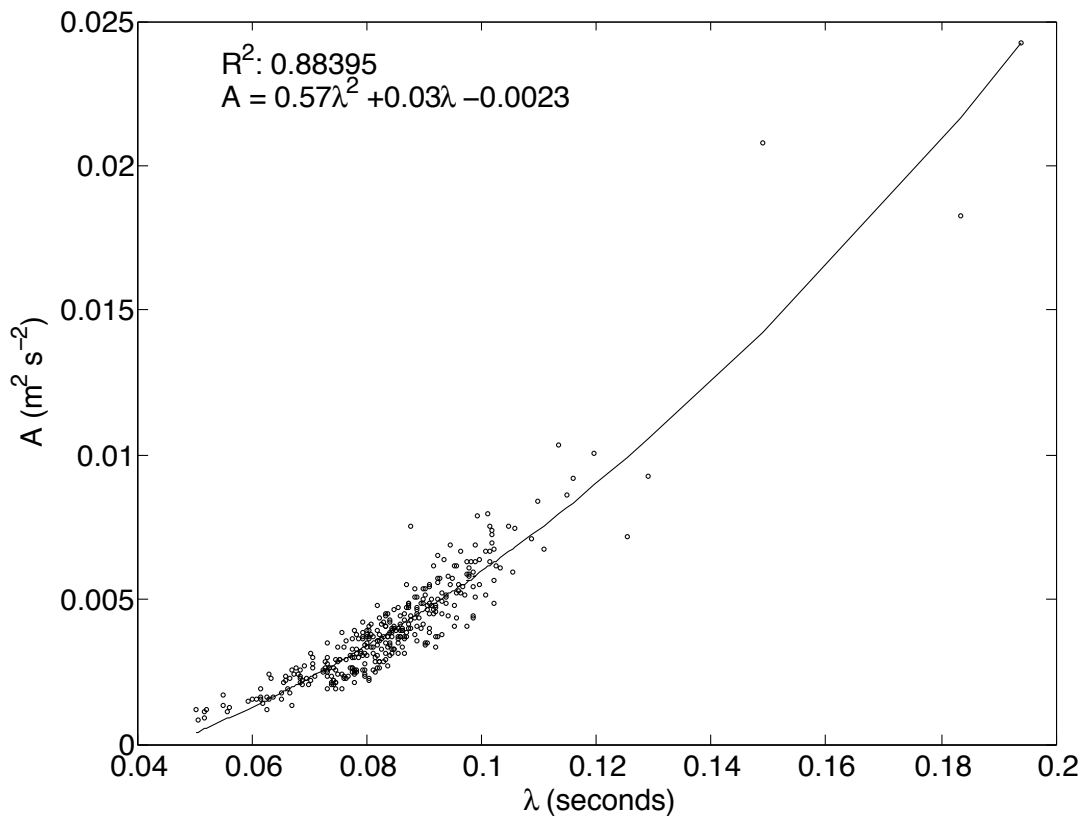

Figure 12: $A$ versus Taylor scale, $\lambda$ for each 10-minute interval with $\bar{u}>$ $0.8 \mathrm{~m} \mathrm{~s}^{-1}$ at Nodule Point with the quadratic fit line and $R^{2}$ value shown. 


\begin{tabular}{c|c|c}
\hline & $\lambda$ & $\Lambda$ \\
\hline \hline$I_{u}$ & 0.596 & 0.450 \\
\hline$T K E$ & 0.747 & 0.079 \\
\hline$\overline{C T K E}$ & 0.680 & 0.017 \\
\hline$A$ & $\mathbf{0 . 8 8 4}$ & 0.317 \\
\hline
\end{tabular}

Table 3: Coefficients of correlation, $R^{2}$, between Taylor scale, $\lambda$, and Integral scale, $\Lambda$, and $I_{u}, T K E, \overline{C T K E}$, and $A$ from the Nodule Point ADV data.

intensity. The four different color schemes on the the barycentric map in Figure 13 highlight the dependencies of each parameter on the anisotropy. The peaks in all four quantities do happen when the flow is furthest from isotropic (three-component limit), but the detailed coloring varies substantially by metric.

The turbulence intensity exhibits a strong relationship with anisotropy, with high $I_{u}$ events approaching the one-component limit. This shows that at this location, the strongest component of the turbulence is aligned in the along-stream direction that is used in the turbulence intensity.

The color based on $\overline{C T K E}$ shows a much weaker relationship with anisotropy, with peaks in $\overline{C T K E}$ scattered throughout the map's domain. The anisotropy magnitude, $A$, is similar to $\overline{C T K E}$, as expected, but with a slightly stronger correlation in predicting the dimensionality of the turbulence. The color based on $T K E$ also shows a relationship between peaks in $T K E$ and onecomponent turbulence, though not as strong a correlation as with $A$. It is interesting to note that as $A$ increases, the turbulence is increasingly 


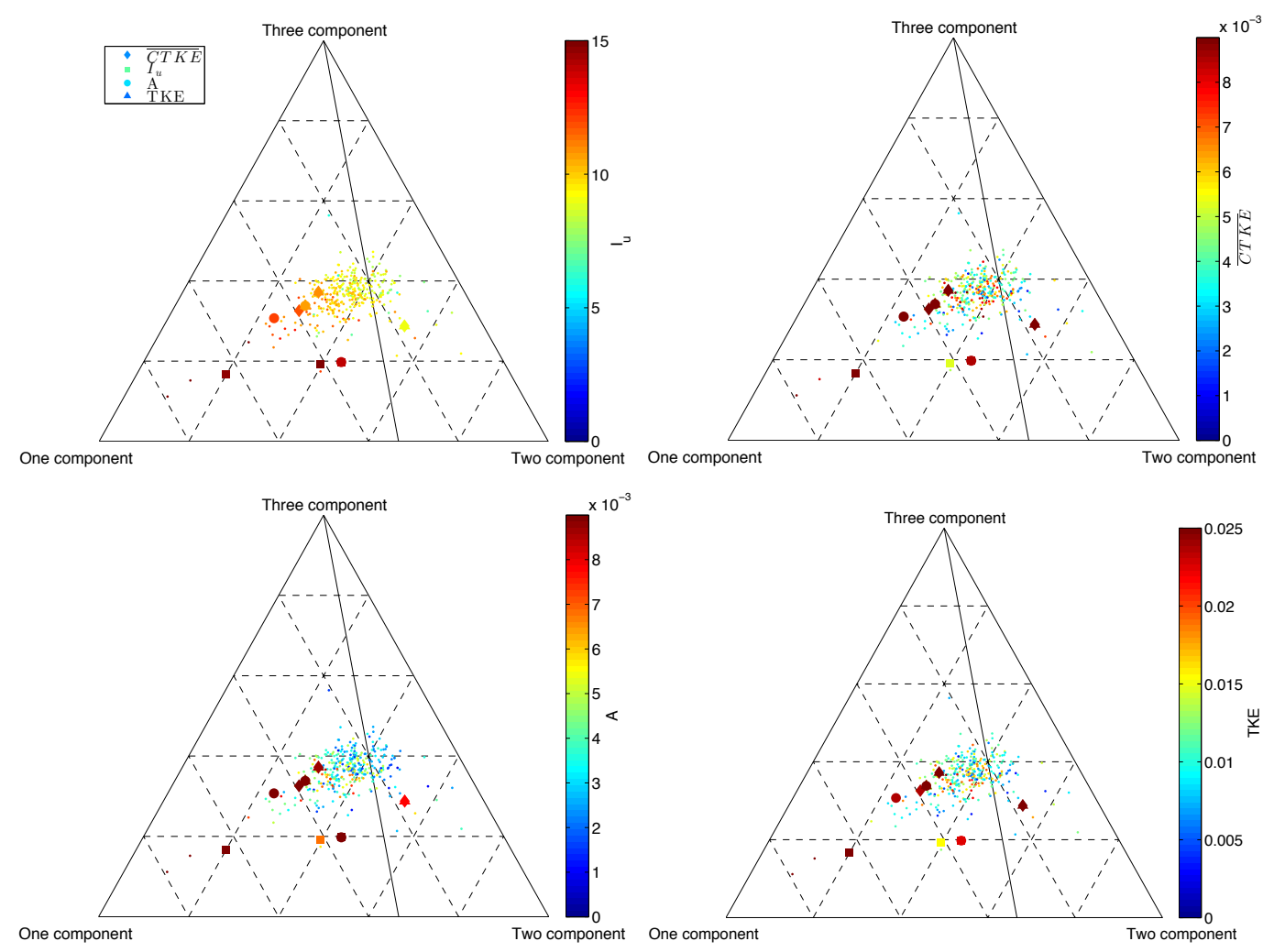

Figure 13: Barycentric maps based on $C_{1 c}, C_{2 c}$, and $C_{3 c}$ for the 10-minute intervals with $\bar{u}>0.8 \mathrm{~m} \mathrm{~s}^{-1}$ at Nodule Point. Coloring is as follows: top left - $I_{u}$; top right - $\overline{C T K E}$; bottom left - A, bottom right - TKE. Three 99thpercentile values of $I_{u}, T K E, \overline{C T K E}$, and $A$ values when $\bar{u}>0.8 \mathrm{~ms}^{-1}$ are shown in the squares, triangles, diamonds, and circles, respectively on each plot, colored according to the map. 
one-dimensional, rather than simply less three-dimensional. This is unexpected, and can be verified with increased observations, and tested in threedimensional models.

The occurrence of one-component "turbulence" may be a sign that some residual of the tidal flow itself continues to be categorized as "turbulence" using 10-minute averages-unfortunately some mixing of mean and turbulence is inevitable when Reynolds averaging is used in turbulent flows without clear scale separation. The time-scale separation analysis in the Appendix aims to minimize this possibility.

Using the comparisons made in this section, it is clear that $A$ captures the behavior of $C T K E$, but provides more physical information than $I_{u}$, correlating closely with both the Taylor correlation scale and the proximity to the most anisotropic corner of the barycentric map. The correlation between $A$ and $C T K E$ can be hypothesized to extend to loads, though direct measurements in a tidal strait are not available yet. These results all support the use of $A$ to parameterize turbulence in a tidal strait, indicating the physical size and shape of turbulence for turbine design and layout purposes (e.g. if anisotropy is planar at length scales of $10 \mathrm{~m}$, then turbines can be sized accordingly). If an additional parameter is to be calculated also, $T K E$ shows the second strongest correlation to length scales, though anisotropy is lacking. The benefit of $T K E$ over $A$ is its previous observations of correlations with decreasing power production. 


\section{Concluding Remarks}

The tidal site of Nodule Point shows strong signs of turbulent events that are expected to impact tidal energy conversion devices. Some of these events have peaks in coherent turbulent kinetic energy, some have peaks in turbulence intensity, and most exhibit one-component anisotropic behavior. The presence of this type of turbulence means that a preferred direction to the loading events is to be expected, putting a particular orientation of strain on the turbine, and violating the common assumption of isotropy. In order to predict these impacts, an analysis of turbulence should include turbulence intensity, either in its traditional or coordinate-system invariant form (the ratio of the full $T K E$ to the mean kinetic energy), and a measure of anisotropy. Both $C T K E$ and $I_{u}$ are useful metrics for measuring turbulence, but when all three velocities are available, the coordinate-independent measures $A, T K E$, and the invariants or eigenvalues of the anisotropy tensor are a preferred physical description that include many details about the directions of the one- and two-components of the turbulence. Other measures of intermittency and coherence, such as the pdfs and structure functions, can be important diagnoses of the degree to which Kolmogorov-like scalings for turbulent cascades hold for the environment of interest. Numerical models that parameterize, rather than directly simulate, the turbulence in similar regions need to be adapted accordingly (e.g., Thyng et al. 2013, Jonkman et al. 2012).

The observed anisotropy at Nodule Point can possibly be attributed to the shallow depth that does not allow isotropy above scales larger than the water depth. In addition, two-dimensional turbulence may be created by 
topographic vortex shedding, which would produce an energy cascade with a slope of $\gamma_{D}=2 / 3$ as was observed here. However, high resolution surface measurements (e.g. SAR, HF radar, or ocean color) are necessary to confirm this hypothesis. Stratification is unlikely to be forcing the two-dimensional flow, since the tidal strait is well-mixed. Therefore, it is predicted that tidal straits in general will have two-dimensional turbulence at scales larger than their depth, although observations at more sites are needed to support this hypothesis.

A next step will compare the statistics of the observations to those of the output of turbulence simulators and turbine models. The observations show strongly that turbulence in this region is not isotropic, even outside of the peaks in turbulence metrics. Turbulence simulators like TurbSim create isotropic turbulence and then add on an optional coherence function [41]. A comparison between the resulting loads and efficiencies for turbines in stochastic turbulence and turbulence created by a physical ocean model (like the Regional Ocean Modeling System of Shchepetkin and McWilliams [54]), or large-eddy simulation (like the National Center for Atmospheric Research LES model of Sullivan et al. [55] and Alexander and Hamlington [56]) for this location will identify the strengths and weaknesses of each type of model in an effort to improve modeling capabilities for turbine design. Thyng et al. [5] compare these ADV records and related observations in this region to a simulation using ROMS. Their results indicate that the large scale flow is adequately simulated, but the subgrid parameterizations in their implementation are unable to fully reproduce the characteristics of the observed turbulence, based on turbulence intensity. They propose ex- 
tensions to these parameterizations that can be used diagnostically and can guide future parameterization improvements. The extensive turbulence characterization performed here provides a more specific explanation of how the parameterizations are currently lacking.

Additional complexity in real-world turbulence at this and similar sites may be hidden in this analysis by the assumption of Taylor's frozen turbulence hypothesis. Several statistics calculated here utilized this hypothesis to estimate spatial information from temporal data. In order to relax that assumption, measurements in time and in space are necessary. An array of ADVs can provide the spatial resolution, improving with the number of locations collecting simultaneous tobservations. Undoubtedly, new insight into the coherent, intermittent in time signals sensed here would result from information about their horizontal and vertical spatial coherence and intermittency.

Acknowledgments Thanks are due to Levi Kilcher of the National Renewable Energy Laboratory for his input. Also, the APL-UW staff for help in field data collection: Joe Talbert, Alex deKlerk, and Capt. Andy ReayEllers. And Brian Polagye (UW) and Marshall Richmond (PNNL) for experiment planning. JT was partially supported by DOE Northwest National Marine Renewable Energy Center. BF-K and PH are partially supported by NSF 1258995,1258907 , and 0934737 . KM is supported by the NOAAESRL/CIRES Graduate Research Fellowship.

\section{Appendix: Time-Scale Separation Analysis}


In decomposing the velocity signal into mean and perturbation, the goal is to capture the small-scale (in time and space) turbulent effects aside from the large-scale (in time and space) tidal effects. Taylor's hypothesis of the relationship between the time and length scales is assumed, which states that for a given turbulent velocity scale, $\hat{u}$, the time and length scales are related as $L / T$. This requires that for turbulence at larger spatial scales, a longer time scale is needed to capture the motions. With a goal of capturing the large, coherent structures in the tidal flow, the largest $\delta t$ possible is desirous. However, the tidal signal impacts the flow at longer time scales, so a careful analysis was needed to separate the two.

The power spectrum of the along stream velocity in Figure .14a shows significant peaks at low frequency, and at tidal frequencies of 2 and 4 cycles per day (diurnal and semi-diurnal tides). Figure .14b shows spectra of the along-stream velocity perturbation obtained through different time windows. With a 60-minute window, the tidal frequencies are still apparent, but with decreasing $\delta t$, the peaks decrease. By $\delta t=5$-minutes, the tidal signal is imperceptible.

Figure .15a shows a 10.5-hour segment of velocities with different $\delta t$ means, and the resulting perturbations with 60,30 , and 20 -minute $\delta t$ are shown in Figure .15b and 10 and 5-minute intervals in Figure .15c. From these plots, it is clear that the 60, 30 and 20-minute averages are too long because a significant linear trend remains over the intervals where the tide is changing direction. That linear trend is therefore being included in the "turbulence," though it is actually the tidal signal. The 10- and 5-minute averages do not show this remaining signal. 

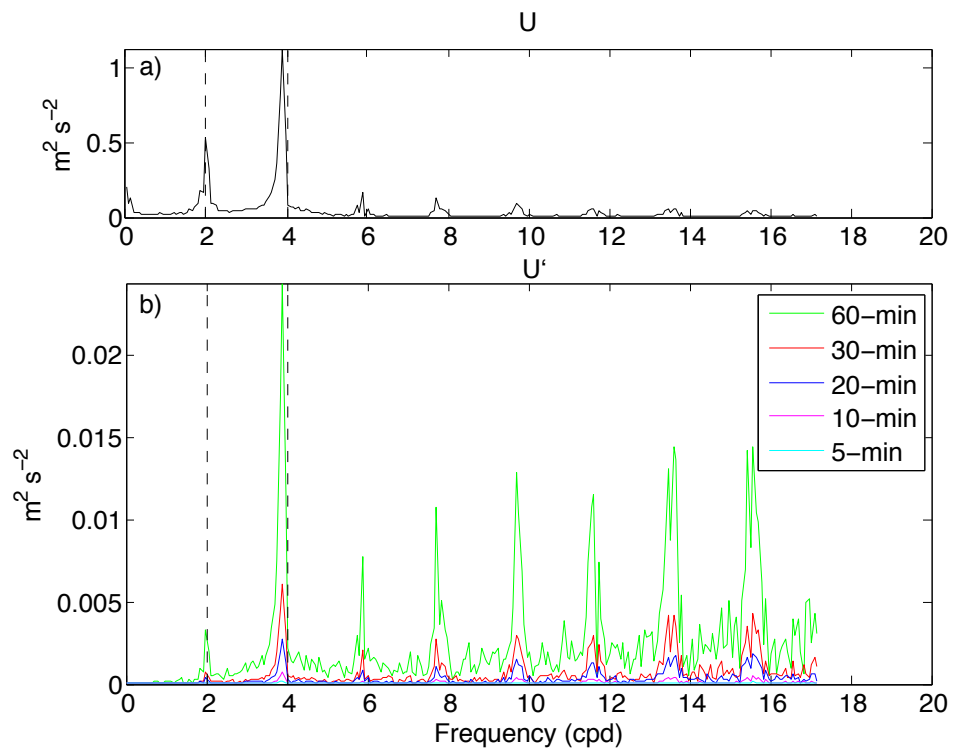

Figure .14: a) Power spectrum of the original velocity signal. b) Spectra of the velocity perturbations for different $\delta t$ values. $\delta t=60$-minute: green, $\delta t=30$-minute: red, $\delta t=20$-minute: blue, $\delta t=10$-minute: magenta, $\delta t=$ 5-minute: cyan 

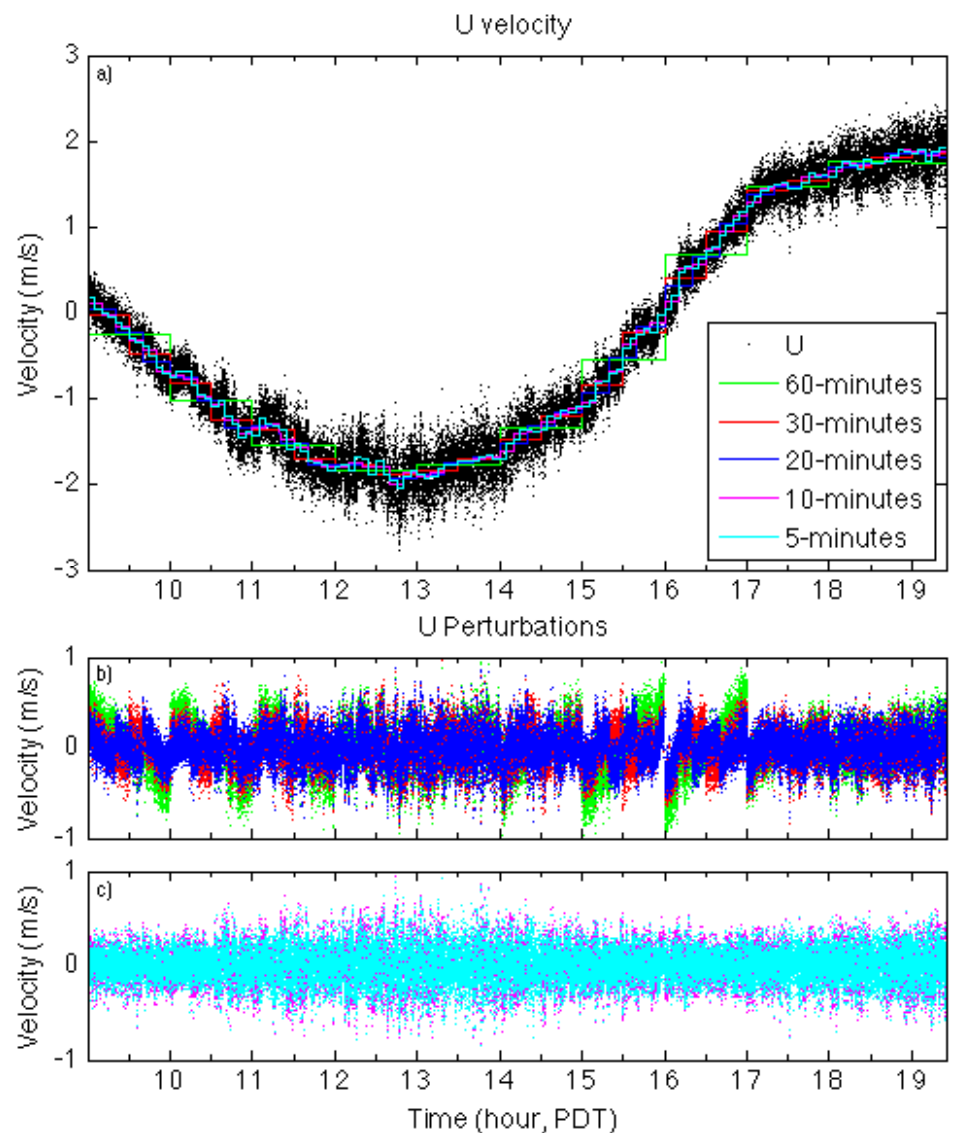

Figure .15: a) Velocity over a 10.5-hour segment with averages shown in color: $\delta t=60$-minute: green, $\delta t=30$-minute: red, $\delta t=20$-minute: blue, $\delta t=10$-minute: magenta, $\delta t=5$-minute: cyan. b) Perturbation from the mean for $\delta t=60$-minute: green, $\delta t=30$-minute: red, and $\delta t=20$-minute: blue. c) Perturbation from the mean for $\delta t=10$-minute: magenta, and $\delta t=$ 5-minute: cyan. 


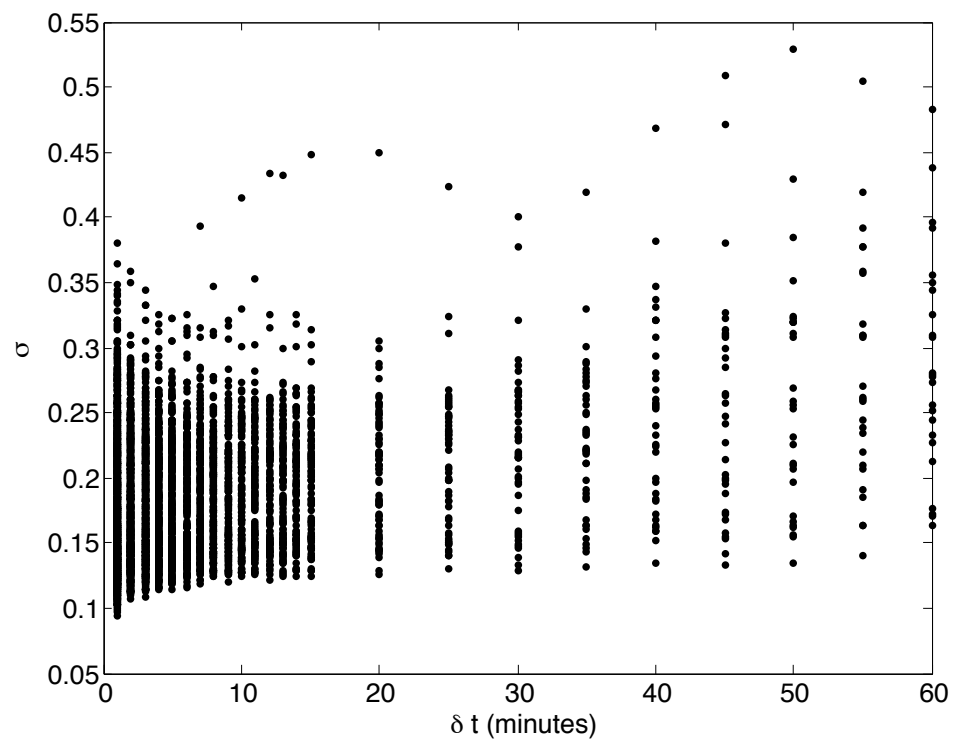

Figure .16: Variances for each interval of along-stream velocity with $\delta t$ varying.

The variance, $\sigma$, for each interval in the entire sample for different $\delta t$ values was computed, and is shown in Figure .16. For the smallest values of $\delta t$, there are higher variances seen, with a decrease as $\delta t$ increases to about $\delta t=5$. Ignoring the outliers, the range of variances stays about constant until approximately $\delta t=35$ when it increases again. Using this range of ideal $\delta t$ values, as well as the information in Figure .15, the higher end of this range is known to be too large. Therefore, after this analysis, the interval length, $\delta t$, for the mean-perturbation decomposition was chosen to be 10 minutes. This captures the largest scales of turbulence without contaminating it with the tidal signal. 


\section{References}

[1] G. I. Taylor, The spectrum of turbulence, Proceedings of the Royal Society of London. Series A-Mathematical and Physical Sciences 164 (1938) 476-490.

[2] G. Taylor, Tidal friction in the irish sea, Philosophical Transactions of the Royal Society of London. Series A, Containing Papers of a Mathematical or Physical Character (1920) 1-33.

[3] H. Grant, R. Stewart, A. Moilliet, Turbulence spectra from a tidal channel, Journal of Fluid Mechanics 12 (1962) 241-268.

[4] A. N. Kolmogorov, The local structure of turbulence in incompressible viscous fluid for very large Reynolds number, Dokl. Akad. Nauk. SSSR $30(1941)$ 9-13.

[5] K. M. Thyng, J. J. Riley, J. Thomson, Inference of turbulence parameters from a ROMS simulation using the $k-\varepsilon$ closure scheme, Ocean Modelling (2013).

[6] J. Jonkman, L. Kilcher, Turbsim user's guide: Version 1.06. 00, Golden, CO: National Renewable Energy Laboratory (2012).

[7] Y. Lu, R. G. Lueck, D. Huang, Turbulence characteristics in a tidal channel, Journal of Physical Oceanography 30 (2000) 855-867.

[8] J. Thomson, B. Polagye, M. Richmond, V. Durgesh, Quantifying turbulence for tidal power applications, Institute of Electrical and Electronics Engineers (2010). 
[9] IEC, Iec 61400-1: Wind turbines part 1: Design requirements, International Electrotechnical Commission (2005).

[10] S. Gooch, J. Thomson, B. Polagye, D. Meggitt, Site characterization for tidal power, in: OCEANS 2009, MTS/IEEE Biloxi - Marine Technology for Our Future: Global and Local Challenges, 2009, pp. 1 -10.

[11] J. Epler, B. Polagye, J. Thomson, Shipboard acoustic Doppler current profiler surveys to assess tidal current resources, in: OCEANS 2010, IEEE, 2010, pp. 1-10.

[12] B. Polagye, J. Thomson, Tidal energy resource characterization: methodology and field study in Admiralty Inlet, Puget Sound, WA (USA), Proceedings of the Institution of Mechanical Engineers, Part A: Journal of Power and Energy 227 (2013) 352-367.

[13] P. Mycek, B. Gaurier, G. Germain, G. Pinon, E. Rivoalen, Experimental study of the turbulence intensity effects on marine current turbines behaviour. part i: One single turbine, Renewable Energy 66 (2014) 729-746.

[14] P. Mycek, B. Gaurier, G. Germain, G. Pinon, E. Rivoalen, Experimental study of the turbulence intensity effects on marine current turbines behaviour. part ii: Two interacting turbines, Renewable Energy 68 (2014) 876-892.

[15] J. Thomson, B. Polagye, M. Richmond, V. Durgesh, Measurements of turbulence at two tidal energy sites in Puget Sound, WA, IEEE Journal of Ocean Engineering 37 (2012) 363-374. 
[16] J. Thomson, L. Kilcher, M. Richmond, J. Talbert, A. deKlerk, B. Polagye, M. Guerra, R. Cienfuegos, Tidal turbulence spectra from a compliant mooring, in: Proceedings of the 1st Marine Energy Technology, Symposium (METS2013), 2013.

[17] B. Gunawan, V. S. Neary, J. Colby, Tidal energy site resource assessment in the East River tidal strait, near Roosevelt Island, New York, New York, Renewable Energy 71 (2014) 509-517.

[18] E. Osalusi, J. Side, R. Harris, Structure of turbulent flow in emec's tidal energy test site, International Communications in Heat and Mass Transfer 36 (2009) 422-431.

[19] K. Korotenko, A. Sentchev, F. G. Schmitt, N. Jouanneau, Variability of turbulent quantities in the tidal bottom boundary layer: Case study in the eastern english channel, Continental Shelf Research 58 (2013) 21-31.

[20] A. Ragheb, M. Ragheb, Wind turbine gearbox technologies, in: Nuclear \& Renewable Energy Conference (INREC), 2010 1st International, IEEE, 2010, pp. 1-8.

[21] A. Monin, On characteristics of an anisotropic turbulence, in: Dokl. Akad. Nauk USSR, volume 75, 1950.

[22] J. G. Charney, Geostrophic turbulence, Journal of the Atmospheric Sciences 28 (1971) 1087-1095.

[23] L. Biferale, I. Procaccia, Anisotropy in turbulent flows and in turbulent transport, Physics reports 414 (2005) 43-164. 
[24] G. Boer, T. Shepherd, Large-scale two-dimensional turbulence in the atmosphere, Journal of the Atmospheric Sciences 40 (1983) 164-184.

[25] C. Frankignoul, Observed interaction between oceanic internal waves and mesoscale eddies, in: Deep Sea Research and Oceanographic Abstracts, volume 23, Elsevier, 1976, pp. 805-820.

[26] X. Capet, J. McWilliams, M. Molemaker, A. Shchepetkin, Mesoscale to submesoscale transition in the california current system. part ii: Frontal processes, Journal of Physical Oceanography 38 (2008) 44-64.

[27] E. Novikov, R. Stewart, The intermittency of turbulence and the spectrum of energy dissipation fluctuations (turbulence intermittency model to calculate spectrum of energy dissipation fluctuations), 1964. (1964).

[28] J. Lumley, G. Newman, The return to isotropy of homogeneous turbulence, Journal of Fluid Mechanics 82 (1977) 161-178.

[29] S. Banerjee, R. Krahl, F. Durst, C. Zenger, Presentation of anisotropy properties of turbulence invariants versus eigenvalue approaches, Journal of Turbulence 8 (2007).

[30] E. A. D'Asaro, J. Thomson, A. Y. Shcherbina, R. R. Harcourt, M. F. Cronin, M. A. Hemer, B. Fox-Kemper, Quantifying upper ocean turbulence driven by surface waves, Geophysical Research Letters 41 (2014) 102-107. URL: http://dx .doi .org/10 . 1002/2013GL058193. doi:10.1002/2013GL058193.

[31] J. A. Polton, S. E. Belcher, Langmuir turbulence and deeply penetrating jets in an unstratified mixed layer, Journal of Geophysical 
Research: Oceans 112 (2007) n/a-n/a. URL: http://dx.doi.org/10. 1029/2007 JC004205. doi:10.1029/2007 JC004205.

[32] A. Tejada-Martinez, C. Grosch, Langmuir turbulence in shallow water. part 2. large-eddy simulation, Journal of Fluid Mechanics 576 (2007) 63-108.

[33] J. Thomson, J. R. Gemmrich, A. T. Jessup, Energy dissipation and the spectral distribution of whitecaps, Geophysical Research Letters 36 (2009).

[34] J. A. Polton, J. A. Smith, J. A. MacKinnon, A. E. Tejada-Martínez, Rapid generation of high-frequency internal waves beneath a wind and wave forced oceanic surface mixed layer, Geophysical Research Letters 35 (2008).

[35] N. Kelley, R. Osgood, J. Bialasiewicz, A. Jakubowski, Using TimeFrequency and Wavelet Analysis to Assess Turbulence/Rotor Interactions, Technical Report, 19th American Society of Mechanical Engineers Wind Energy Symposium, 2000.

[36] ISO 4354, Wind Actions on Structures, 1997.

[37] D. L. Elliott, J. Cadogan, Effects of wind shear and turbulence on wind turbine power curves, Technical Report, Pacific Northwest Lab., Richland, WA (USA), 1990.

[38] J. MacEnri, M. Reed, T. Thiringer, Influence of tidal parameters on seagen flicker performance, Philosophical Transactions of the Royal So- 
ciety A: Mathematical, Physical and Engineering Sciences 371 (2013) 20120247.

[39] S. Wharton, J. K. Lundquist, Atmospheric stability affects wind turbine power collection, Environmental Research Letters 7 (2012) 014005.

[40] T. Mücke, D. Kleinhans, J. Peinke, Atmospheric turbulence and its influence on the alternating loads on wind turbines, Wind Energy 14 (2011) 301-316.

[41] B. Jonkman, TurbSim User's Guide: Version 1.50, Technical Report, National Renewable Energy Laboratory, 2009.

[42] N. Kelley, B. Jonkman, G. Scott, J. Bialasiewicz, L. Redmond, The Impact of Coherent Turbulence on Wind Turbine Aeroelastic Response and Its Simulation, Technical Report, National Renewable Energy Laboratory WindPower Conference, 2005.

[43] F. Maganga, G. Germain, J. King, G. Pinon, E. Rivoalen, Experimental characterisation of flow effects on marine current turbine behaviour and on its wake properties, IET Renewable Power Generation 4 (2010) 498509.

[44] B. Gaurier, P. Davies, A. Deuff, G. Germain, Flume tank characterization of marine current turbine blade behaviour under current and wave loading, Renewable Energy 59 (2013) 1-12.

[45] S. B. Pope, Turbulent flows, Cambridge University Press, Cambridge, 2000. URL: http://www.loc.gov/catdir/description/cam0210/ 99044583.html. 
[46] E. K. Webb, Ratio of spectrum and structure-function constants in the inertial subrange, Quarterly Journal of the Royal Meteorological Society 90 (1964) 344-345.

[47] P. Wiles, T. Rippeth, J. Simpson, P. Hendricks, A novel technique for measuring the rate of turbulent dissipation in the marine environment, Geophysical Research Letters 33 (2006).

[48] N. Lucas, S. J.H., T. Rippeth, C. Old, Measuring turbulent dissipation using a tethered ADCP, Journal of Physical Oceanography (2013) submitted.

[49] K. McCaffrey, B. Fox-Kemper, G. Forget, Estimates of ocean macroturbulence: Structure function and spectral slope from argo profiling floats, Journal of Physical Oceanography (in revision).

[50] R. H. Kraichnan, Inertial ranges in two-dimensional turbulence, Physics of Fluids 16 (1967) 1417-1423.

[51] U. Frisch, Turbulence: The Legacy of A. N. Kolmogorov, Cambridge University Press, Cambridge, 1995.

[52] J. Lumley, Computational modeling of turbulent flows, Advances in Applied Mechanics 18 (1978) 123-176.

[53] A. Cayley, A memoir on the theory of matrices, Philosophical transactions of the Royal society of London 148 (1858) 17-37.

[54] A. F. Shchepetkin, J. C. McWilliams, The regional oceanic model- 
ing system (ROMS): a split-explicit, free-surface, topography-followingcoordinate oceanic model, Ocean Modelling 9 (2005) 347-404.

[55] P. P. Sullivan, J. C. McWilliams, C.-H. Moeng, A subgrid-scale model for large-eddy simulation of planetary boundary-layer flows, BoundaryLayer Meteorology 71 (1994) 247-276.

[56] S. R. Alexander, P. E. Hamlington, Study of turbulence statistics in large eddy simulations of ocean current turbine environments, in: Proceedings of the ASME 2014 33rd International Conference on Ocean, Offshore and Arctic Engineering, ASME, 2014. 


\section{a) U velocity}

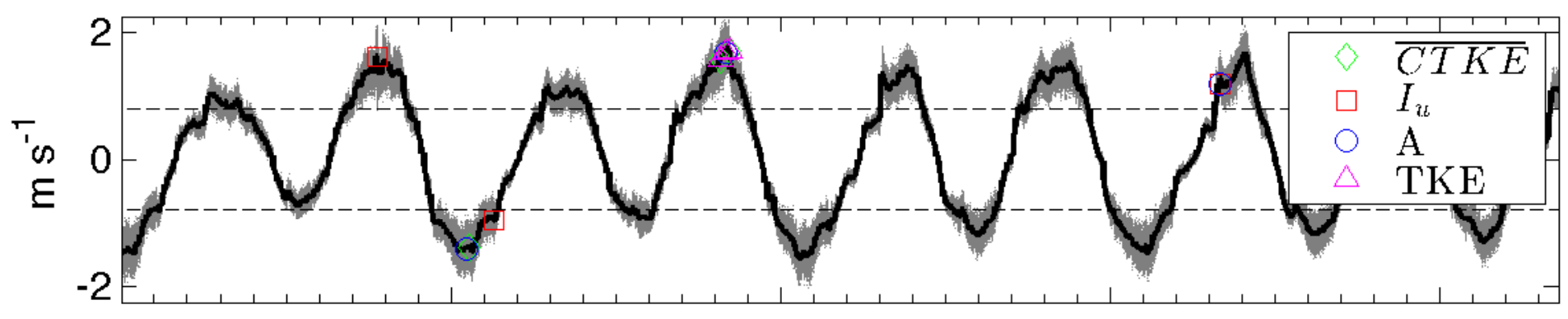

b) $\vee$ velocity

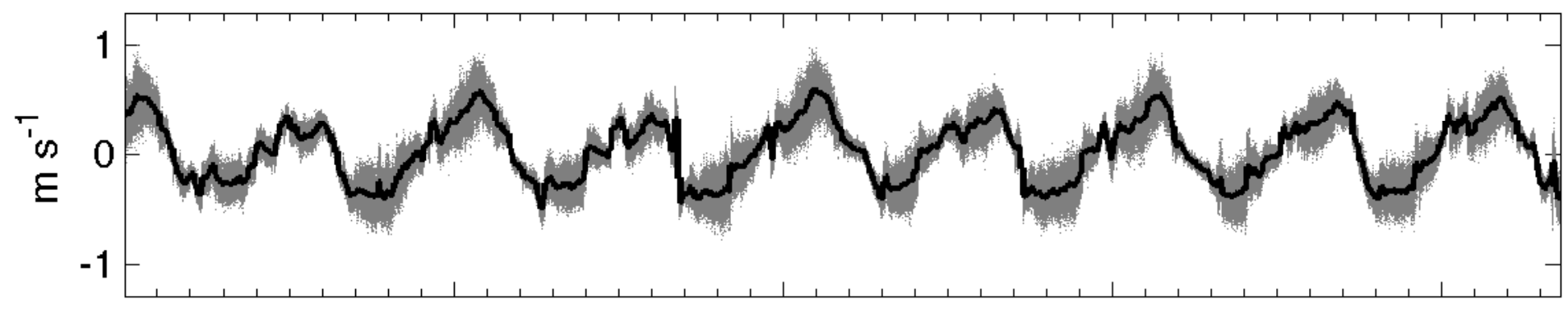

c) W velocity

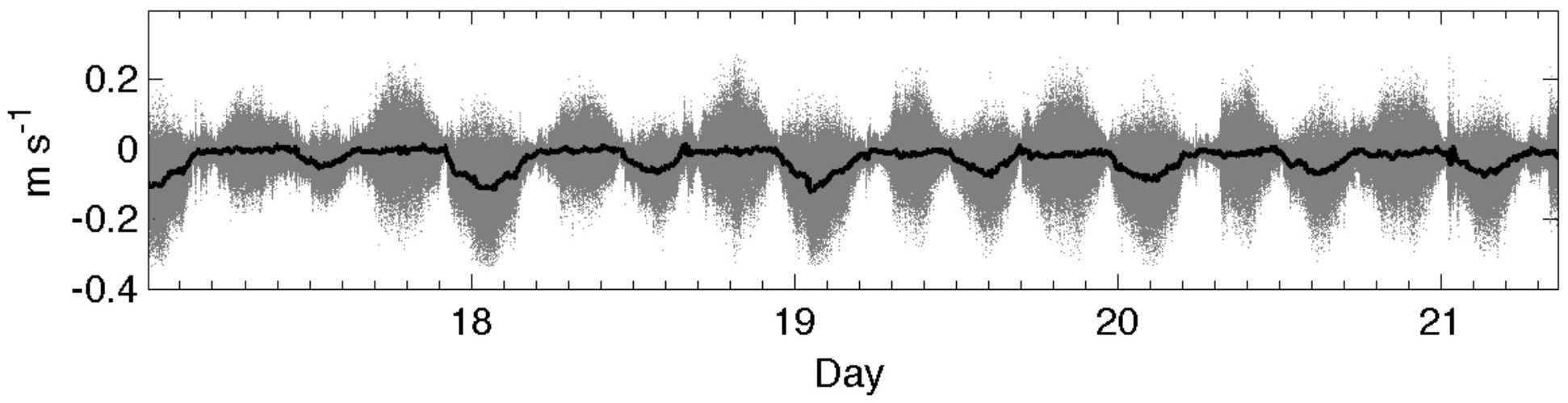


a) Velocity Perturbations

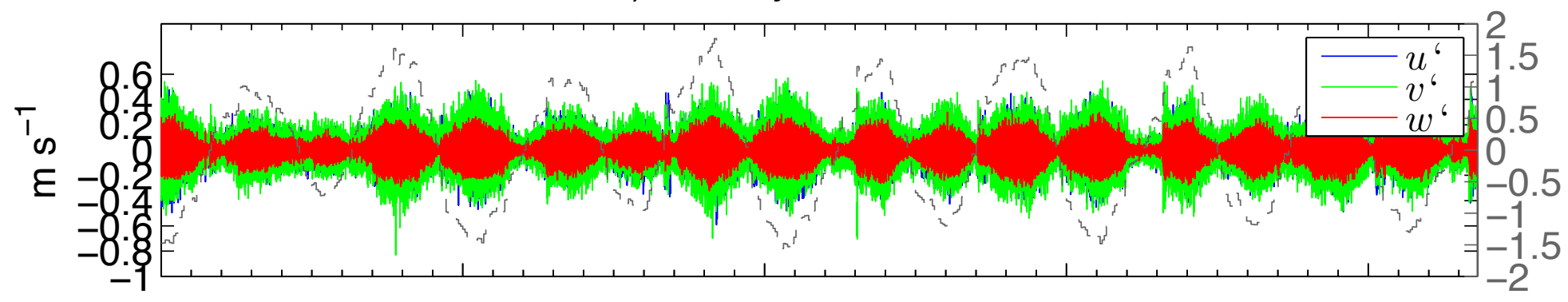

b) Normal Stresses

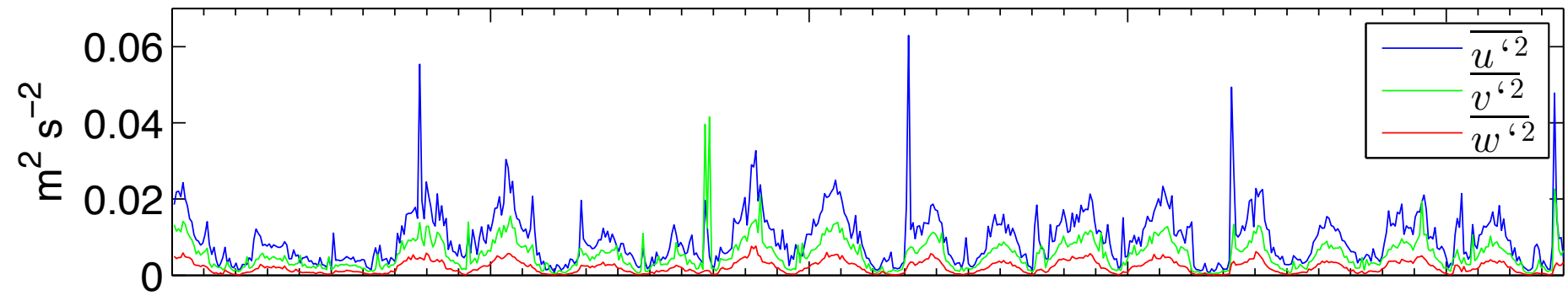

c) Shear Stresses

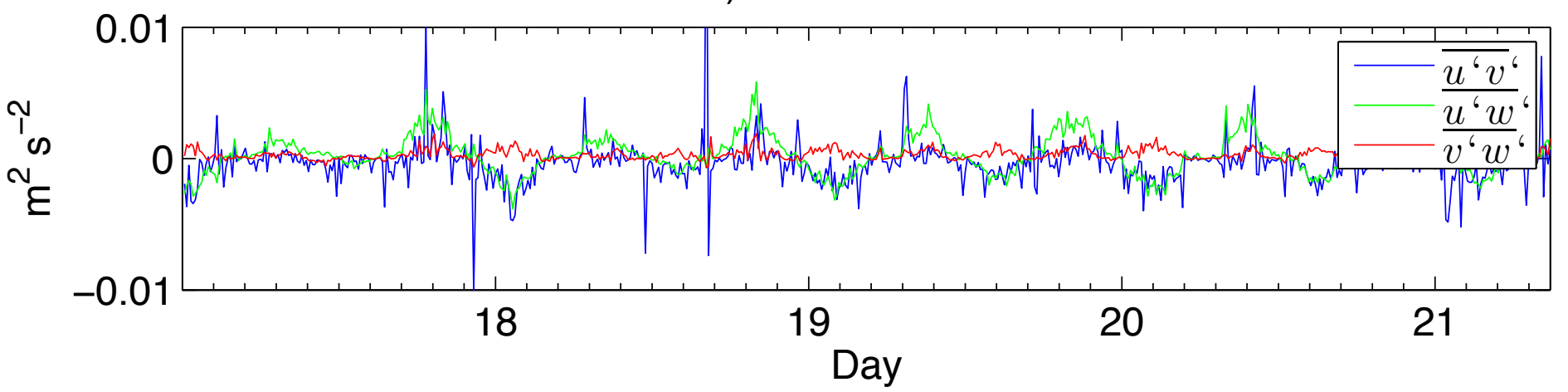


a) $I_{u}$

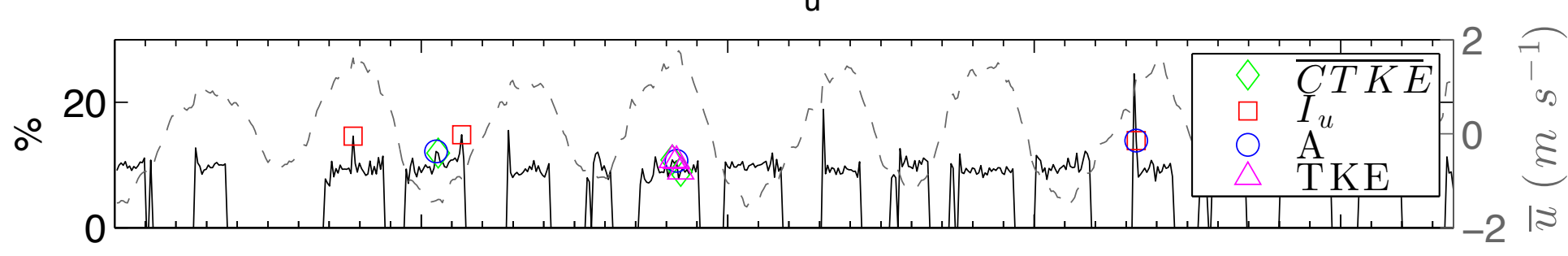

b) TKE

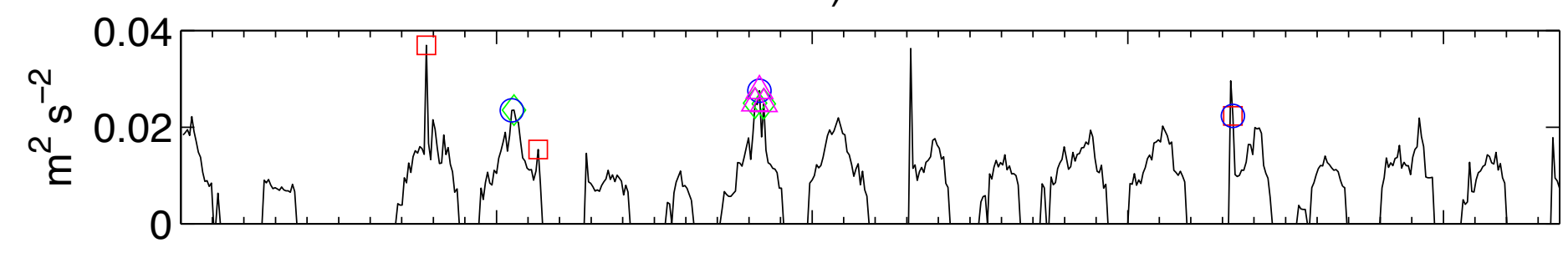

c) $\overline{C T K E}$
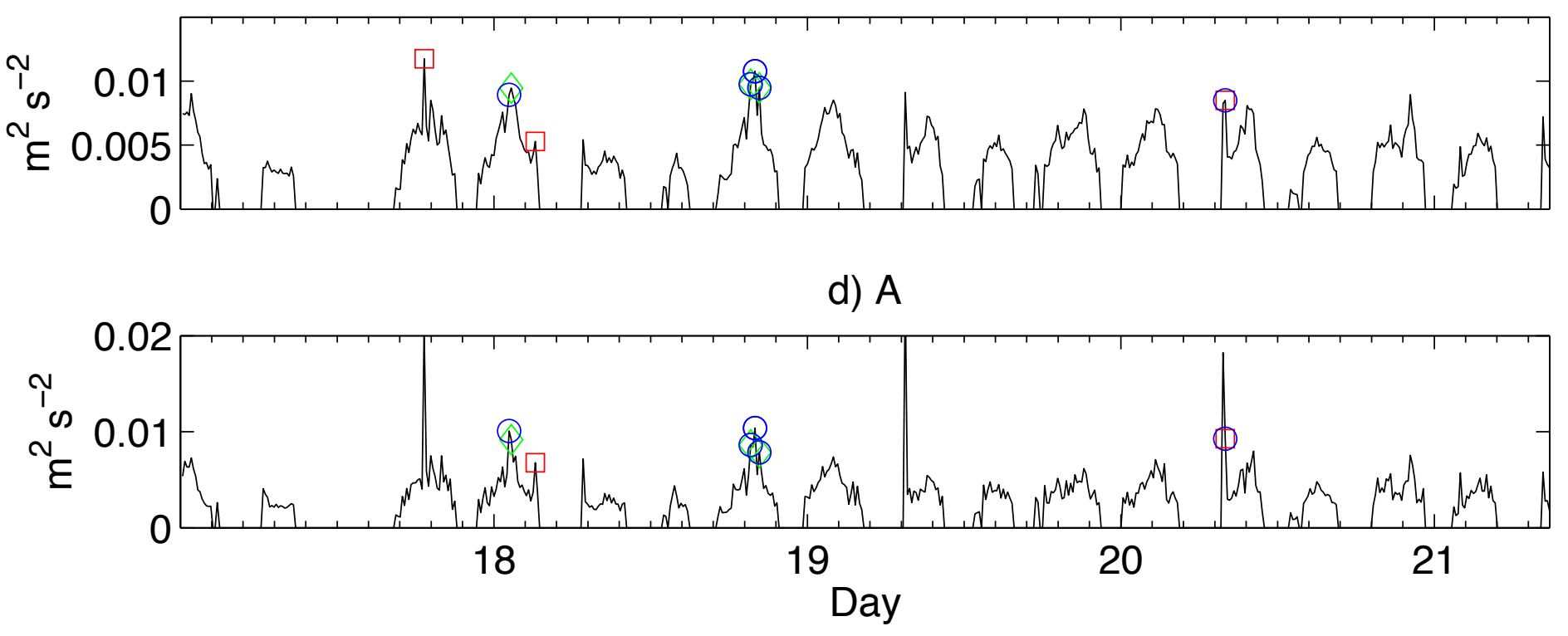


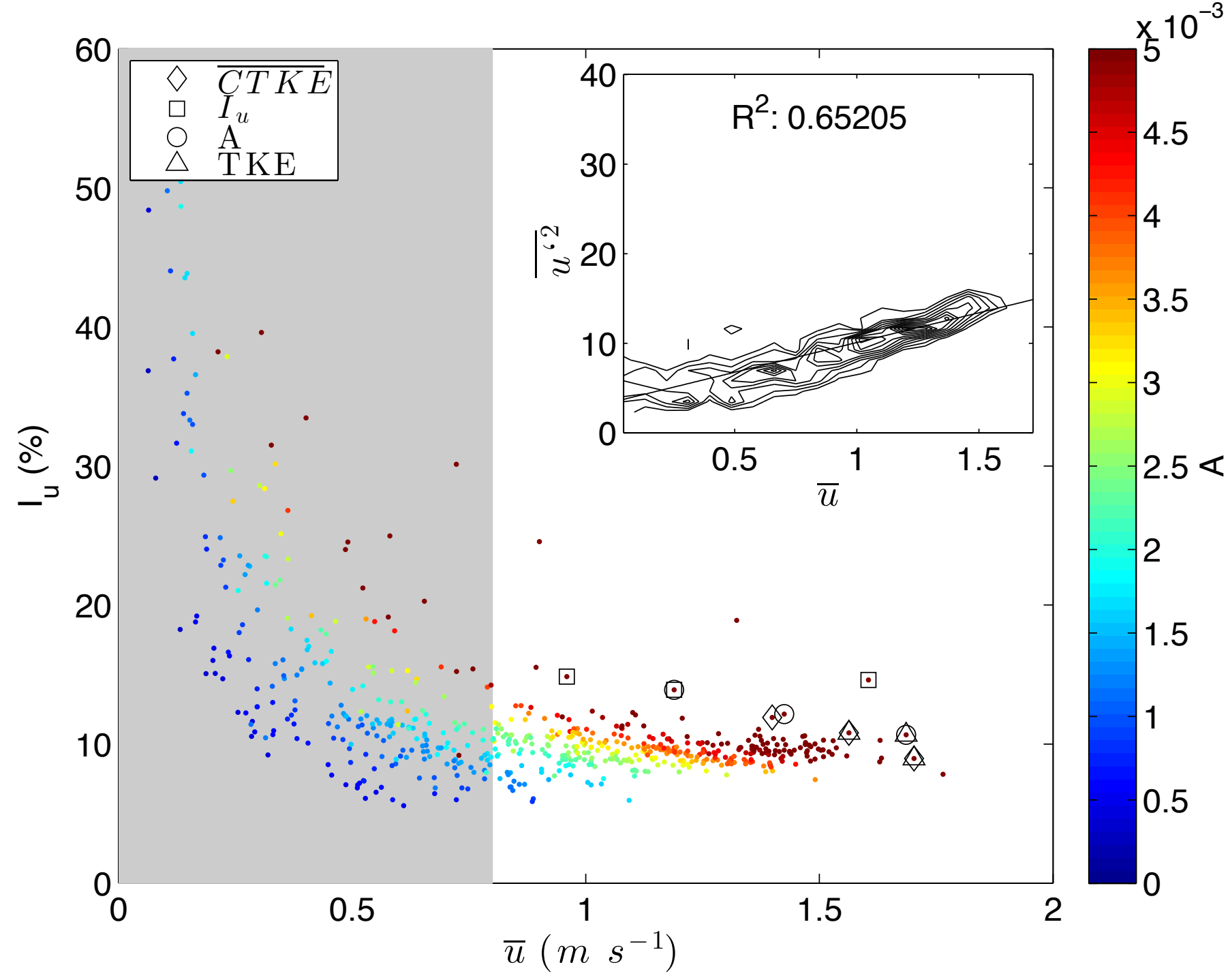




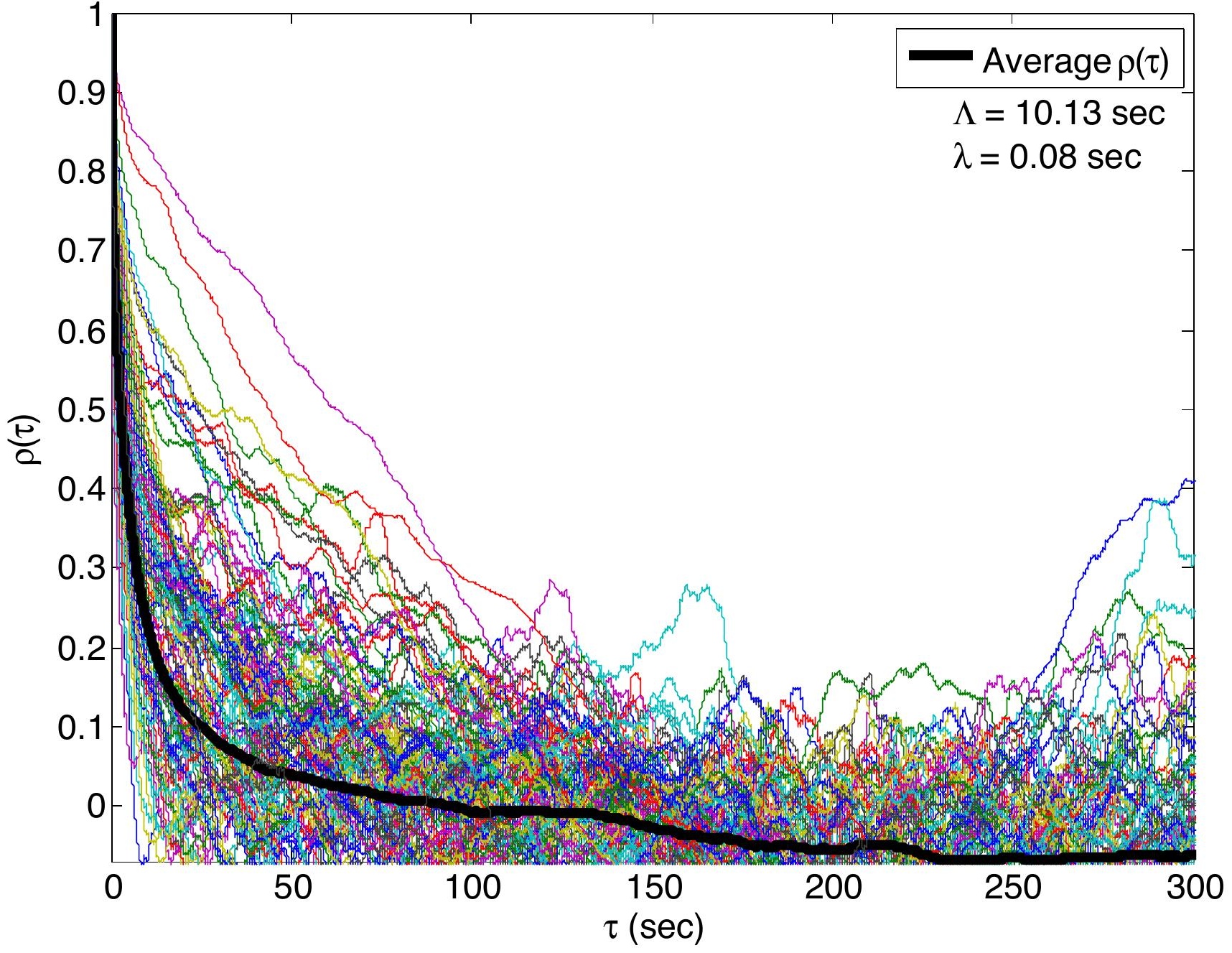




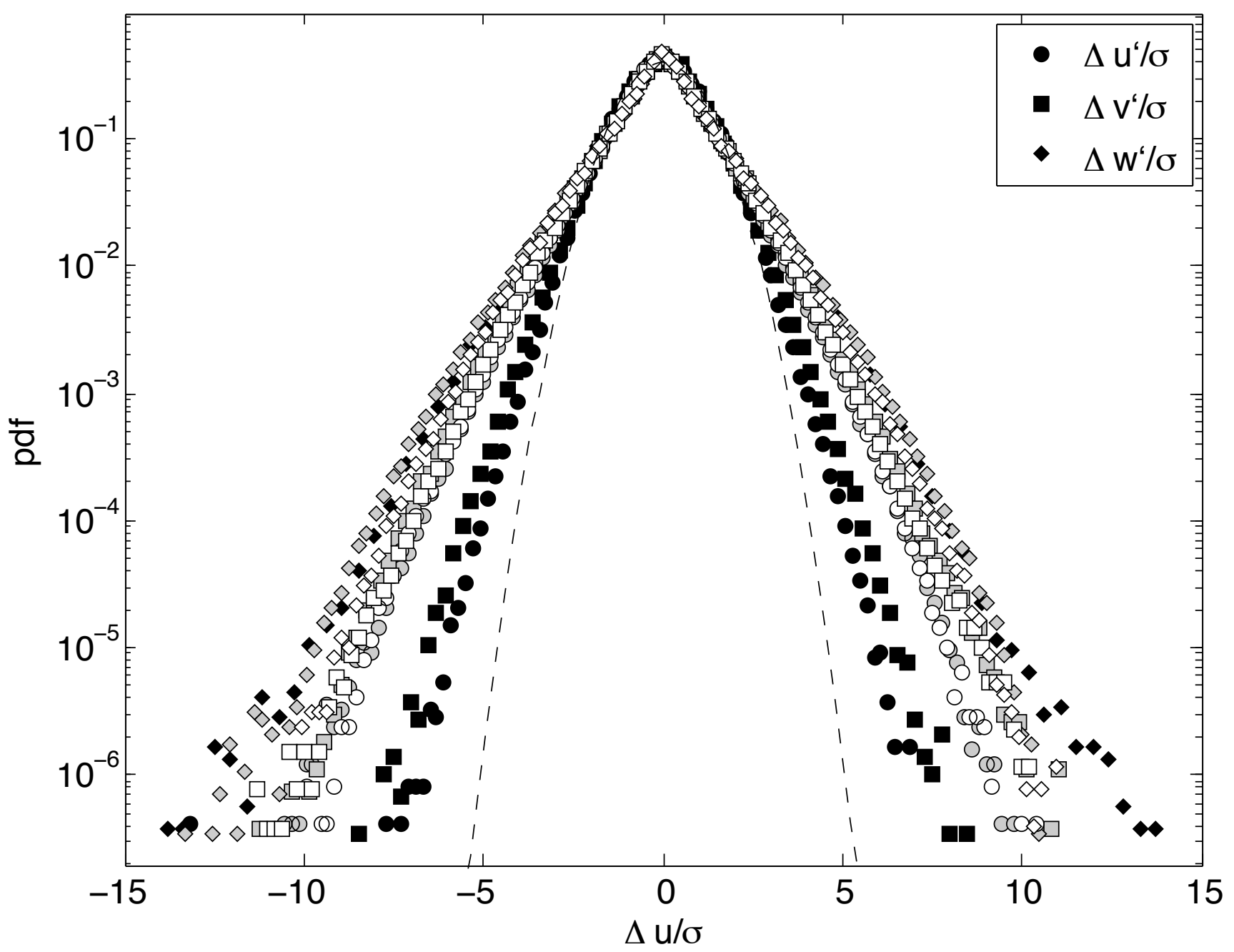




\section{Mean}

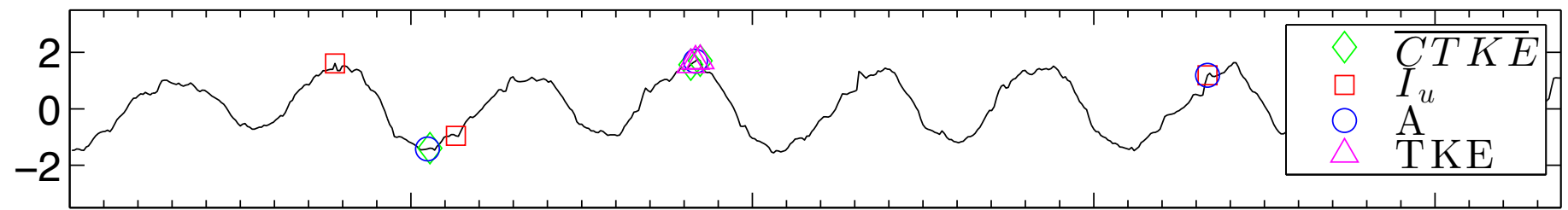

\section{Standard Deviation}

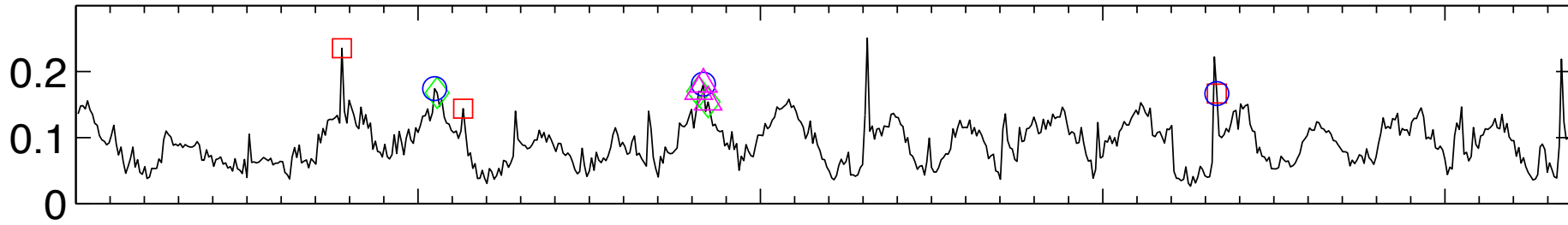

\section{Skewness}

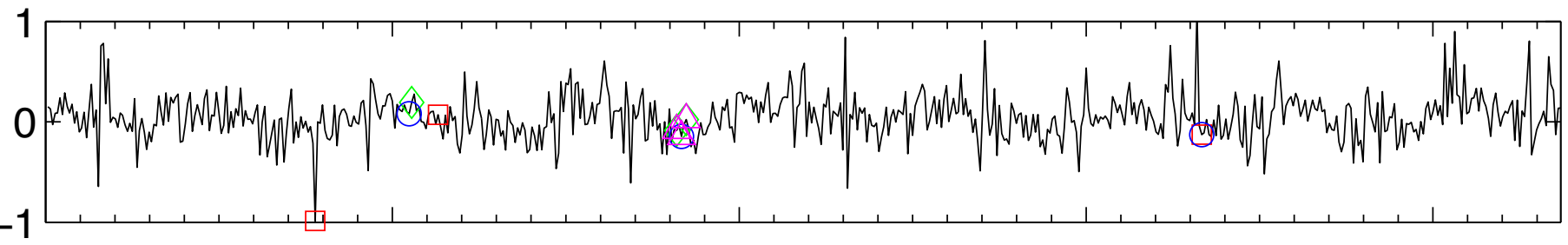

\section{Kurtosis}

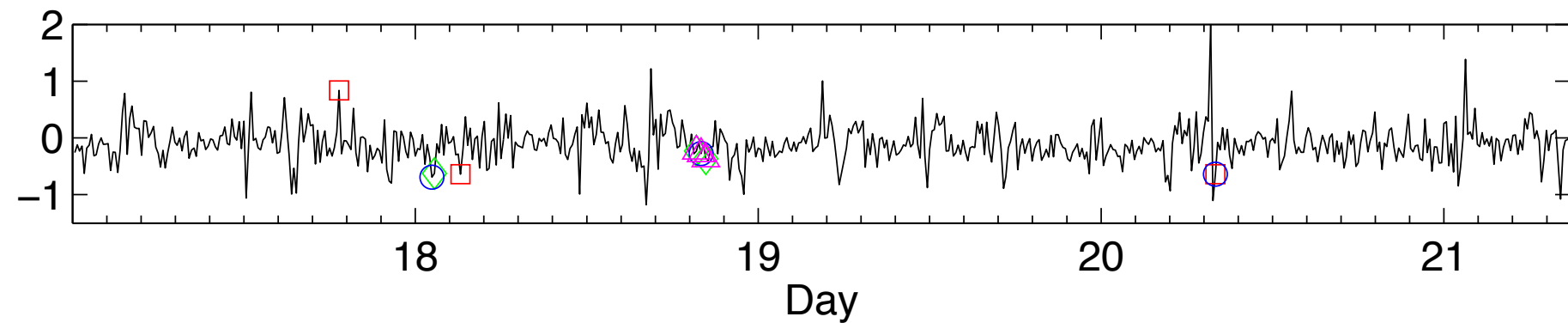



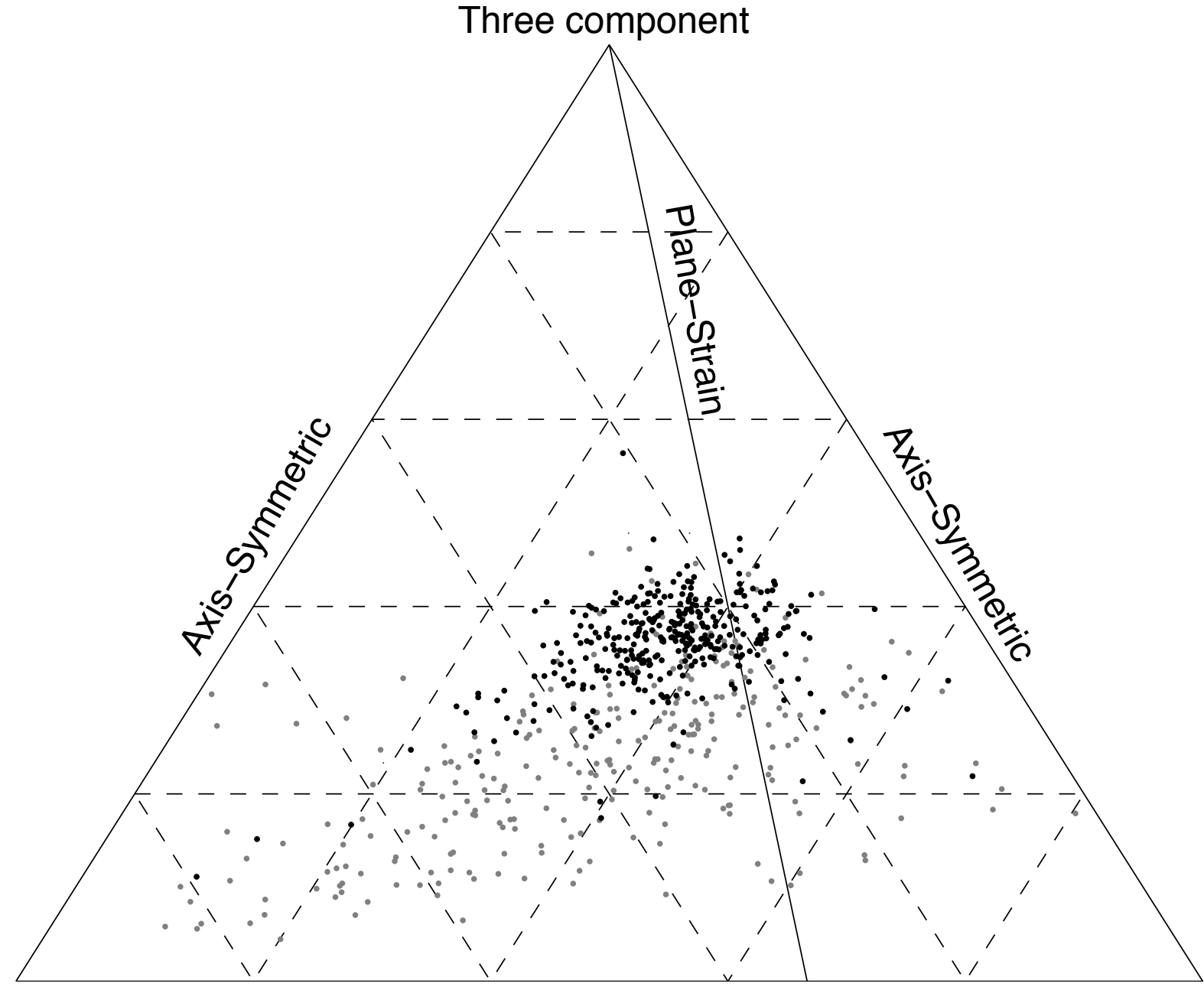


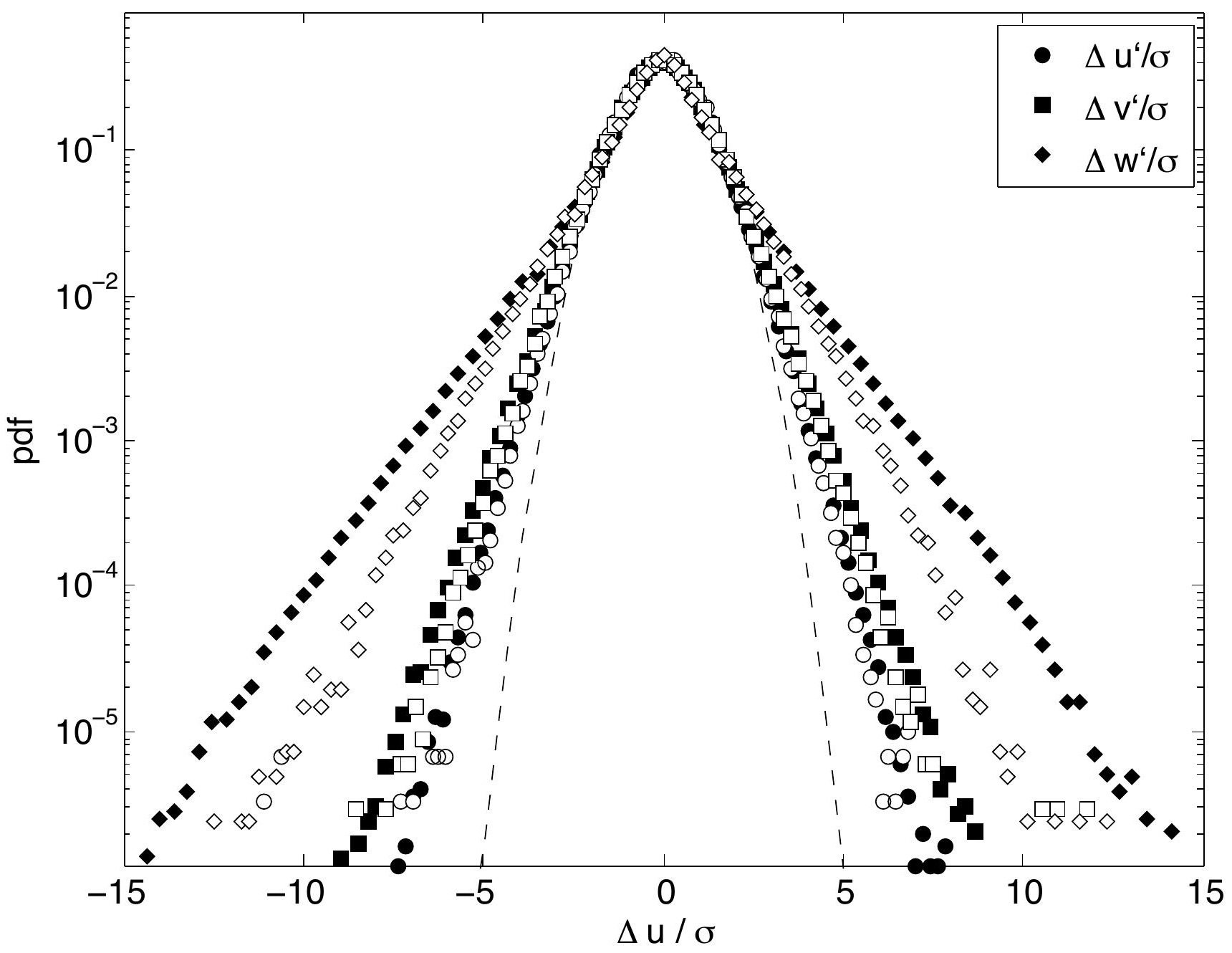




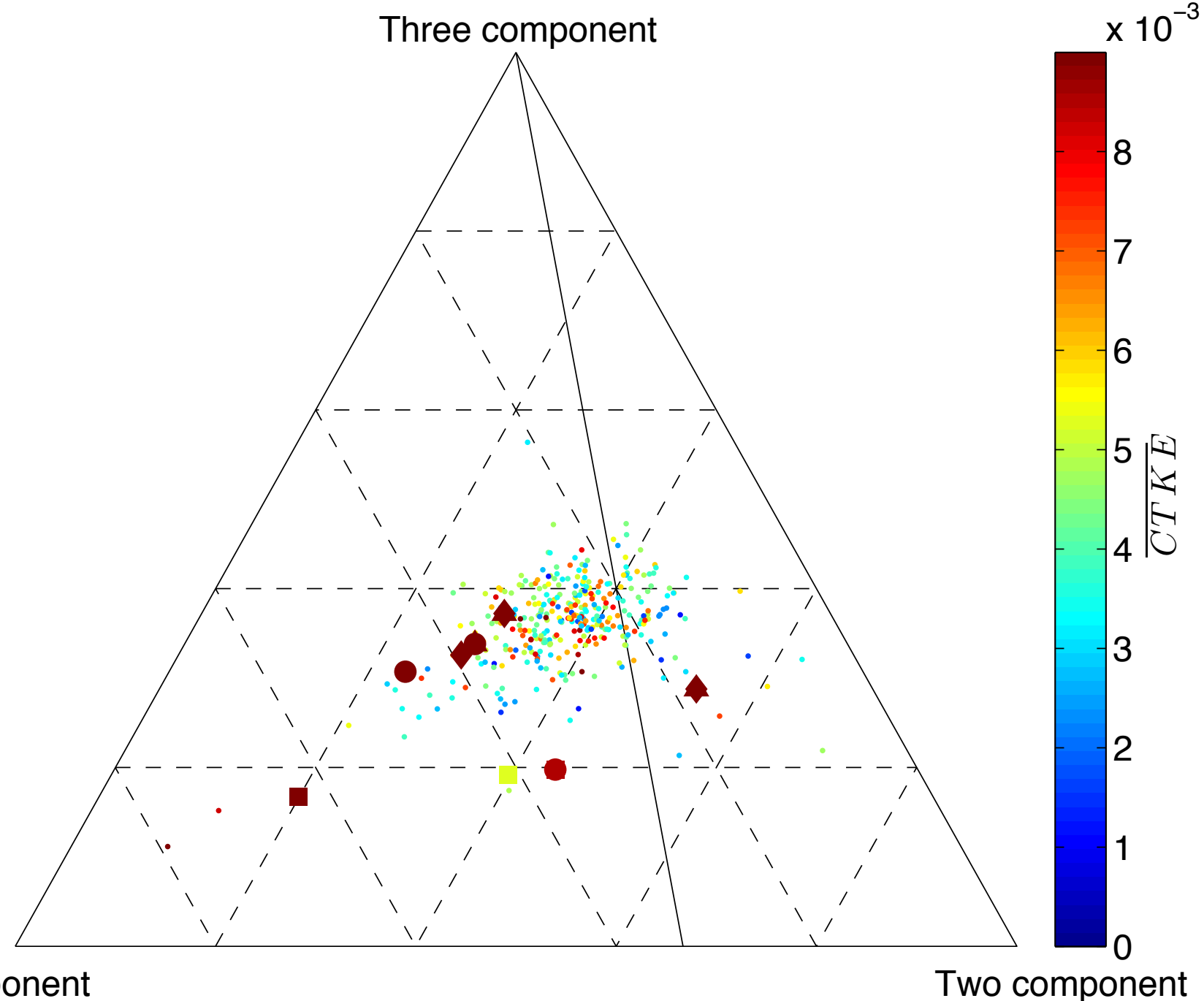




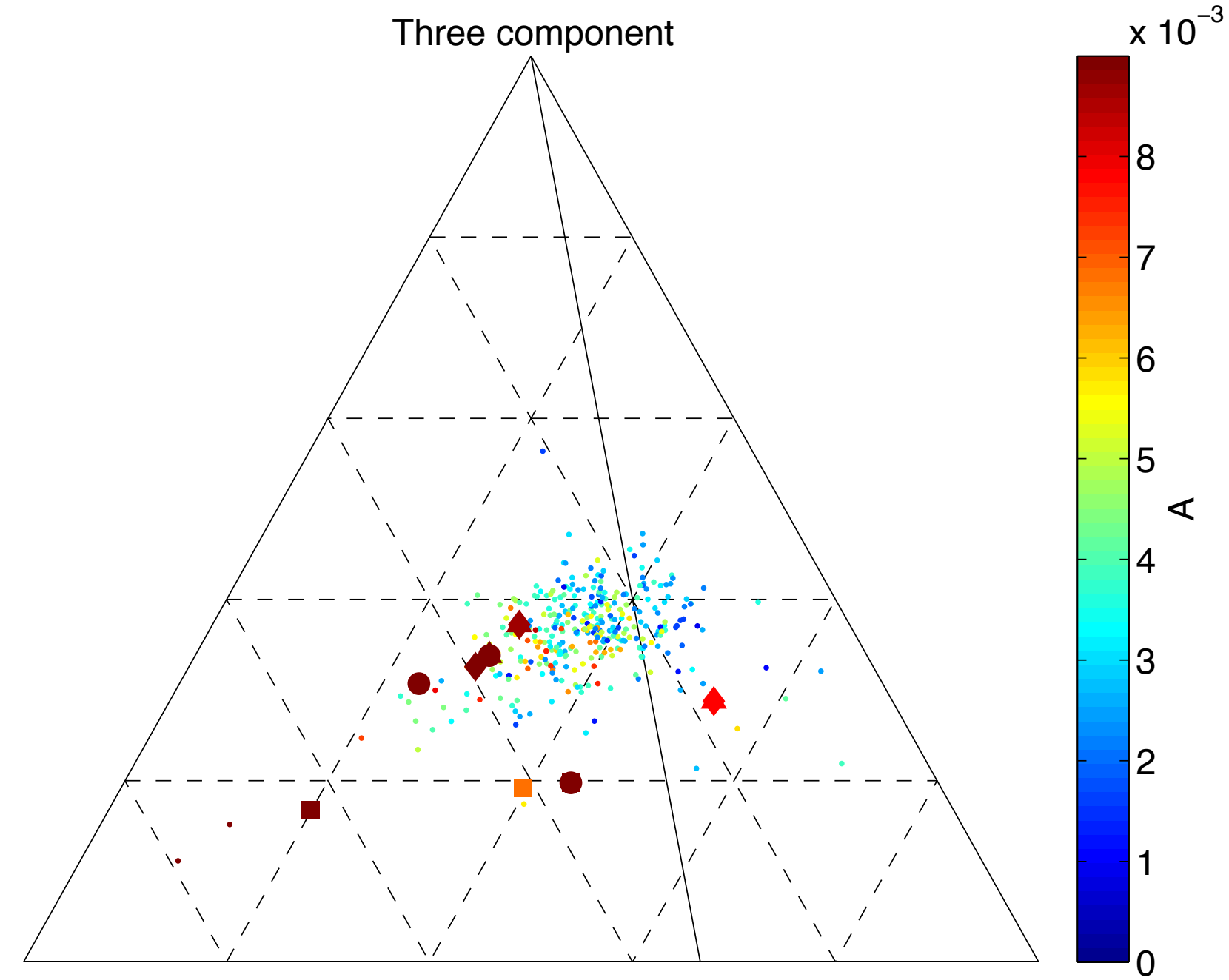




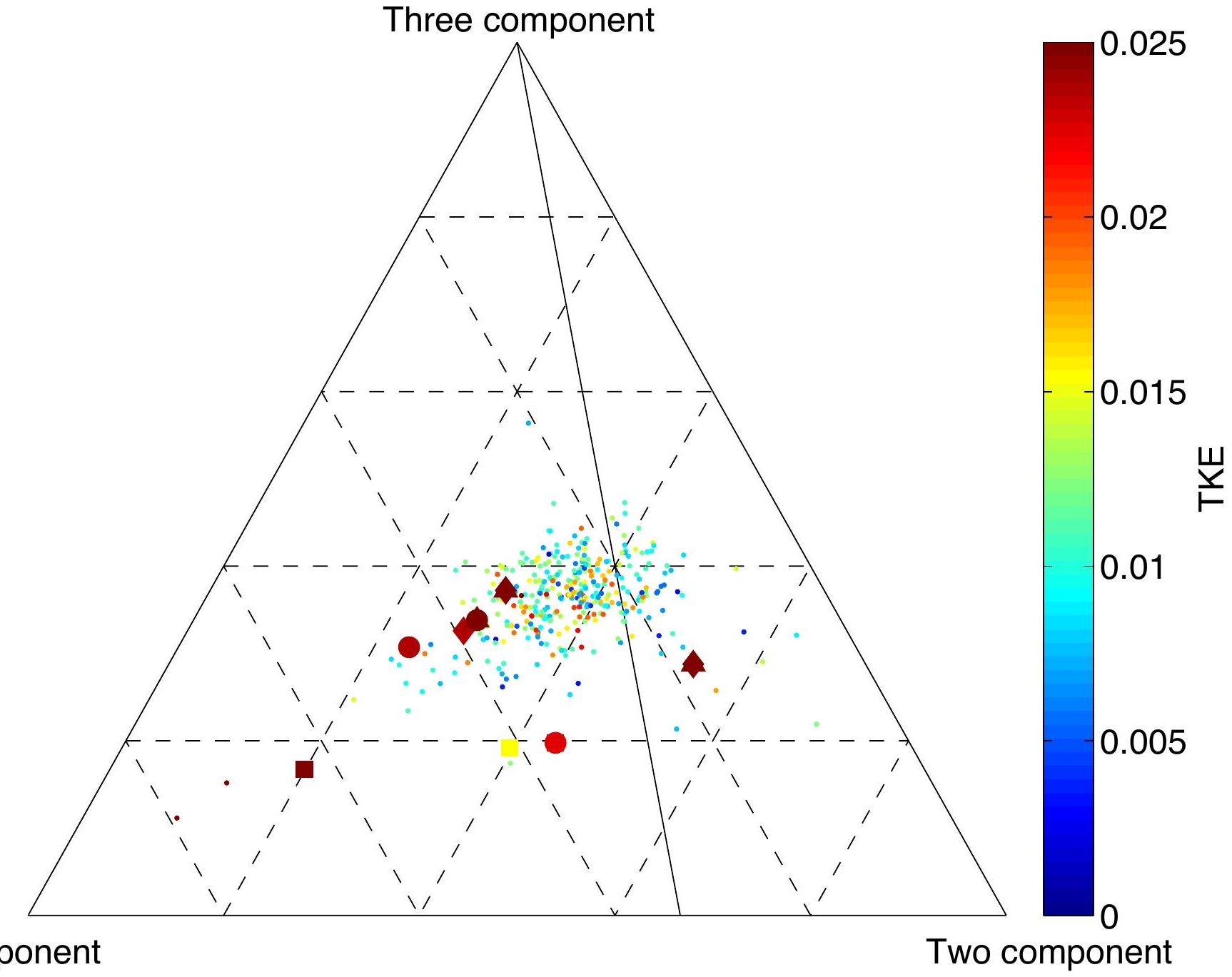


U

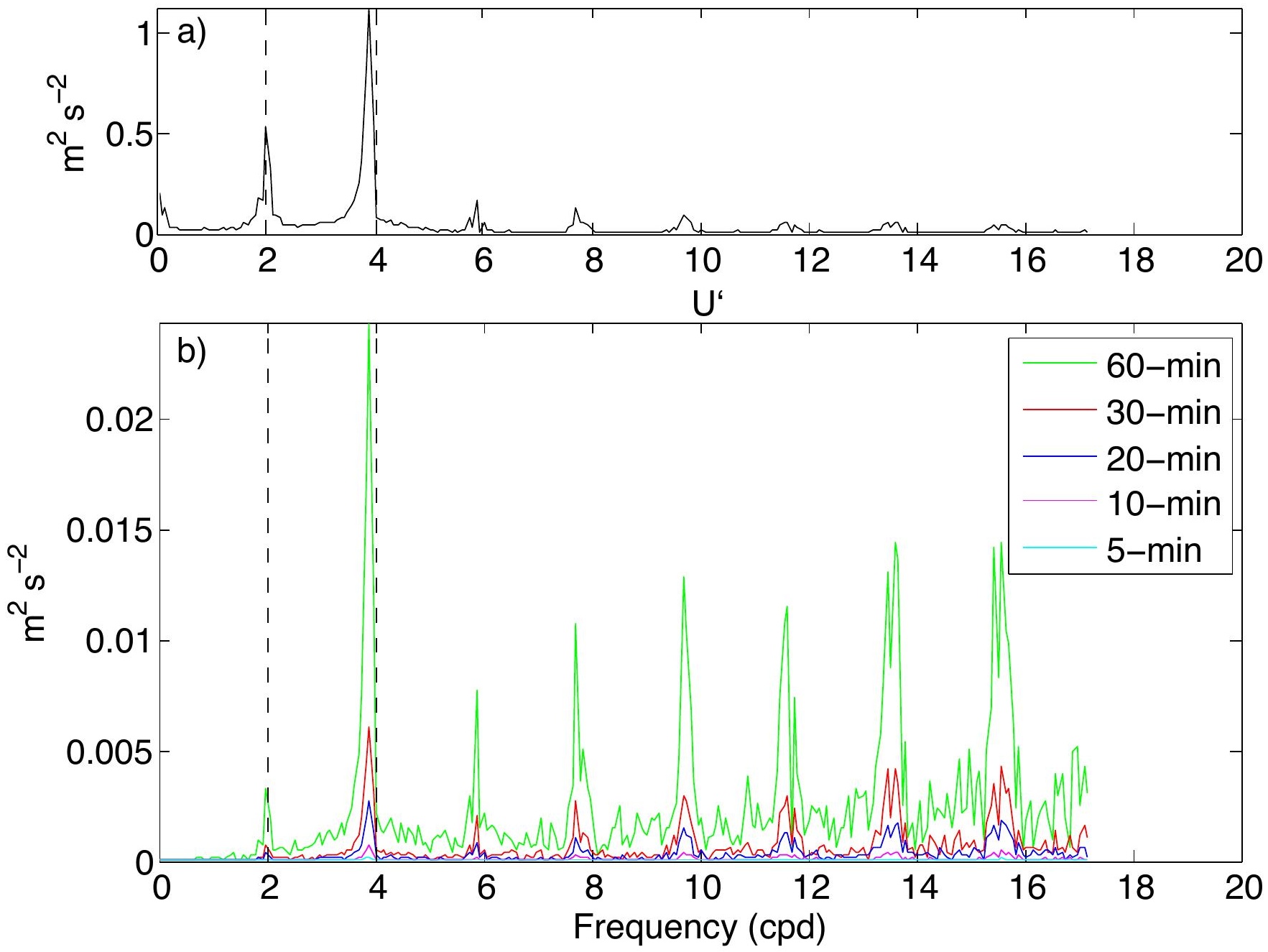




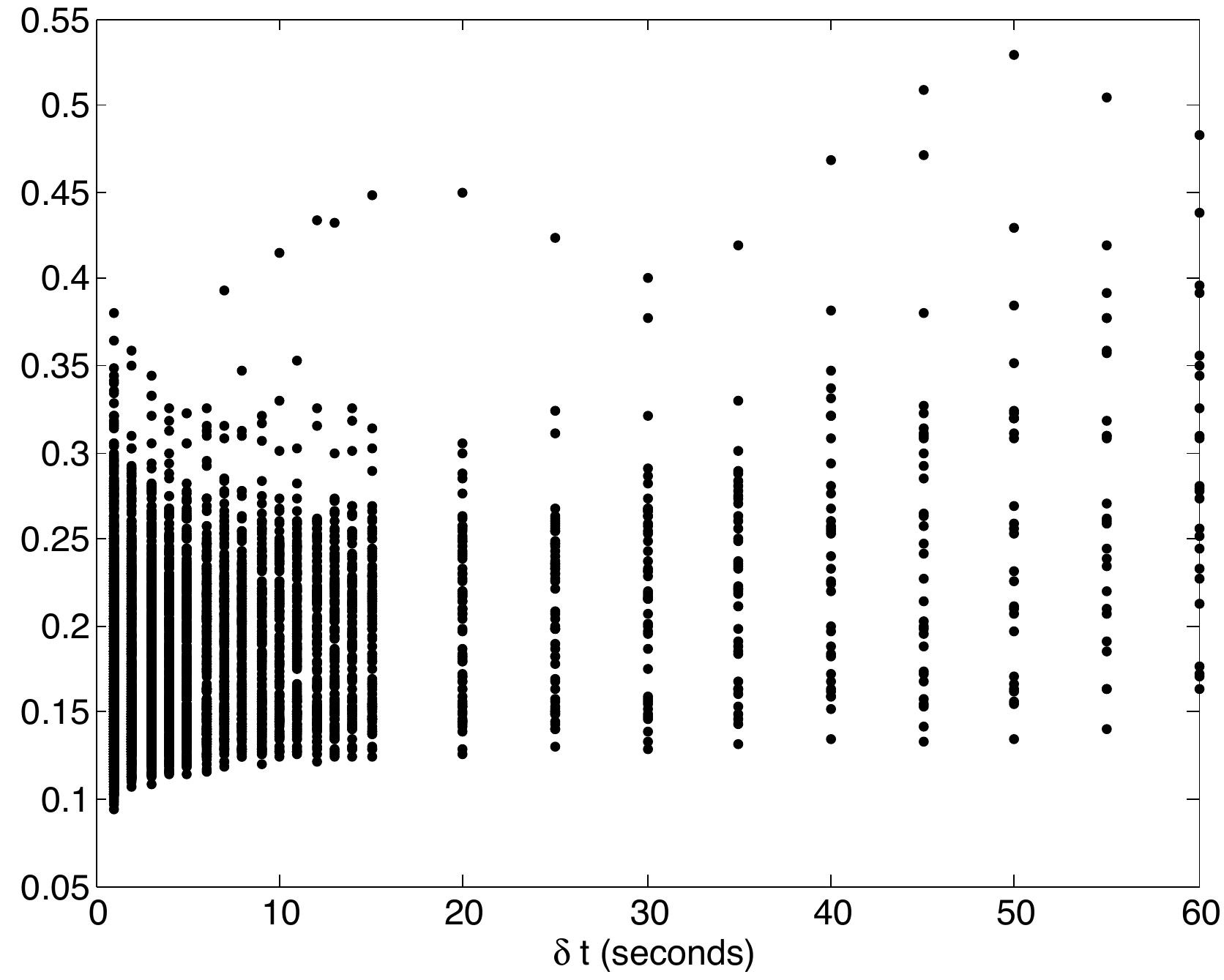

